\title{
EIGENVALUES BELOW THE ESSENTIAL SPECTRA OF SINGULAR ELLIPTIC OPERATORS
}

\author{
W. D. EVANS AND ROGER T. LEWIS ${ }^{1}$
}

\begin{abstract}
A new technique is developed for determining if the number of eigenvalues below the essential spectrum of a singular elliptic differential operator is finite. A method is given for establishing lower bounds for the least point of the essential spectrum in terms of the behavior of the coefficients and weight near the singularities. Higher-order operators are included in these results as well as second-order Schrödinger operators.
\end{abstract}

1. Introduction. In this paper we are concerned with the qualitative theory of the discrete spectrum below the essential spectrum for elliptic partial differential operators which are bounded below. We develop a new technique for determining if the number of eigenvalues below the essential spectrum is finite as well as estimating the least point of the essential spectrum $l_{e}$. Cases of arbitrary order are treated in a unified manner as well as more extensive and improved results being given in the second-order case.

Briefly, the mathematical interest in the problem arises from separation of variables and the subsequent desire for eigenfunction expansions. In nonrelativisitic quantum mechanical systems the eigenvalues below the essential spectrum, which is itself typically an interval $\left[l_{e}, \infty\right)$, have associated eigenfunctions which are "bounded" states of the system since a particle in such a state cannot leave the system without additional energy. In the case of general vibrating systems, which may correspond to higher order operators, the eigenvalues below the essential spectrum are associated with standing waves. General references for these types of studies are the books of Reed and Simon [23, 24] and Schechter [26, 28].

Some of the most noted attention which the problem of the finiteness of the eigenvalues below $l_{e}$ has received is that of Allegretto [3-7], Piepenbrink [19-21], Moss and Piepenbrink [16], and more recently Schmincke [30]. For a general discussion of some of this work and its significance, we refer the reader to the survey article of Simon [31]. These papers have been primarily concerned with the problem when the only singularity is at infinity, which is not the case in many important physical problems. Attempts to extend the results to higher-order operators have not

Received by the editors September 9, 1985. Presented at the Sixth Czechoslovak Conference on Differential Equations (EQUADIFF 6), Brno, August 1985. Sponsored by the Czechoslovak Mathematical Society.

1980 Mathematics Subject Classification. Primary 35P15; Secondary 35J25, 47B25, 47F05.

${ }^{1}$ This author was partly supported by a National Science Foundation grant number DMS- 8503331 . 
been successful except in very special cases (see [5]). Using an alternative to the "nonoscillation condition" of these papers, we have established a new condition for finiteness of the bound states which simplifies the requirements on the weight and coefficients, which includes the case of finite and infinite singularities, and which applies as well to quite general, higher-order, selfadjoint differential operators.

The specific problem of determining if the number of bound states of an $N$-body quantum system is finite or infinite is what partly motivates these investigations. The paper of Simon [31] and the notes in Reed and Simon [23, 24] are excellent places to begin tracking these particular studies.

An important part of a study such as this is to estimate the least point $l_{e}$ of the essential spectrum. Much of the earlier work on this problem has been from the point of view of perturbation theory. We wish to deal with the situations which cannot necessarily be approached directly from a perturbation theory point of view. For related work we refer to work of Persson [18], Schechter [27, 28], and [9]. The methods used here are principally those established earlier by Edmunds and Evans [8]. For specific results concerning $l_{e}$ in relation to $N$-body quantum systems we again recommend to the reader the book of Read and Simon [24] and, in particular, the HVZ Theorem (named for W. Hunziker, C. Van Winter, and G. Zhislin) contained therein.

2. The domain $\Omega$. The boundary-value problem to be considered is defined on a connected open subset (i.e., a domain) $\Omega$ of $\mathbf{R}^{n}, n \geqslant 1$, which may be either bounded or unbounded. If $\Omega$ is unbounded then $\infty$ is considered to be on the boundary of $\Omega$ in the sense of a one point compactification of $\mathbf{R}^{n}$. We denote the finite points of its boundary by $\partial \Omega$ and closure by $\bar{\Omega}$. The boundary conditions to be imposed on $\partial \Omega$ involve normal derivatives, and our requirements will be met if we assume that outside some singular set $S$, in which the conditions are not necessarily met, $\partial \Omega$ not only has a normal but is also smooth enough for the usual embedding theorems to hold. In this way we can accommodate boundaries $\partial \Omega$ with nasty kinks of arbitrary severity, such points or subsets of $\partial \Omega$ being included in the set $S$. Points in $S \cap \partial \Omega$ also include those values of $s \in \partial \Omega$ where the coefficients or weight of the operator fail to satisfy the requirements presented below. To be more specific we require that $S \backslash\{\infty\}$ be a closed subset of $\partial \Omega$. Let $\Gamma_{S} \equiv N_{S} \cap \partial \Omega$ and $\Gamma_{R} \equiv \partial \Omega \backslash \Gamma_{S}$, where $N_{S}$ is an open neighborhood of $S \backslash\{\infty\}$ in $\mathbf{R}^{n}$. If $\Omega$ is unbounded then $\{\infty\} \subseteq S$; in fact, $S=\{\infty\}$ is allowed when $\Omega$ is unbounded.

Before giving the aforementioned smoothness requirements on $\partial \Omega \backslash S$ we first need some notation and background information. Partial derivatives are written in terms of

$$
D^{\alpha}=D_{1}^{\alpha_{1}} \cdots D_{n}^{\alpha_{n}}, \quad D_{i}^{\alpha_{i}}=\left(\frac{\partial}{\partial x_{i}}\right)^{\alpha_{i}}
$$

where $\alpha_{1}, \ldots, \alpha_{n}$ are nonnegative integers and $\alpha=\left(\alpha_{1}, \ldots, \alpha_{n}\right)$ is a multi-index of order $|\alpha|=\alpha_{1}+\alpha_{1}+\cdots+\alpha_{n}$. For $m \in \mathbf{N}$, the Sobolev space $W^{m, 2}(\Omega)$ consists of the set of complex-valued functions $u$ : $\Omega \rightarrow \mathrm{C}$ with distributional derivatives $D^{\alpha} u \in$ 
$L^{2}(\Omega)$ for $|\alpha| \leqslant m$ and the norm

$$
\|u\|_{m, \Omega}=\left\{\sum_{0 \leqslant|\alpha| \leqslant m} \int_{\Omega}\left|D^{\alpha} u\right|^{2}\right\}^{1 / 2} ;
$$

when $m=0, W^{0,2}(\Omega)=L^{2}(\Omega)$, and we denote its norm by $\|u\|_{\Omega}$.

Let $G$ be a bounded open set in $\mathbf{R}^{n}$. Following Fraenkel [10] we define $\partial G$ to be of class $C^{m}, m \in \mathbf{N}$, if (a) $\partial G=\partial \bar{G}$ and (b) for each point $a \in \partial G$ there exists an open neighborhood $U(a)$ of $a$ in $\mathbf{R}^{n}$, local coordinates $y^{\prime}=\left(y_{1}, \ldots, y_{n-1}\right)$ and $y_{n}$ with $y=\left(y^{\prime}, y_{n}\right)=0$ at $x=a$ and a function $h=h(\cdot, a)$ such that $\partial G \cap U(a)$ has a representation

$$
y_{n}=h\left(y^{\prime}\right), \quad y^{\prime} \in \mathscr{C}(a), \quad h \in C^{m}(\overline{\mathscr{C}}(a) ; \mathbf{R})
$$

where $\mathscr{C}(a)$ is a convex open neighborhood of 0 in $\mathbf{R}^{n-1}$ (cf. Definition 9.2 in [2, p. 128]).

We refer the reader to Fraenkel's paper for the merits of this smoothness criterion and a comparison with the multitude of conditions which abound in the literature. Note that Fraenkel does not require $G$ to be bounded as we assume; for a bounded $G, \partial G$ is of class $C^{m}$ if and only if it is uniformly of class $C^{m}$ according to Definition 3.4 in [10], (see [10, Theorem 3.5]).

If $G$ is a bounded domain with $\partial G$ of class $C^{m}$ the set of restrictions to $\Omega$ of functions in $C_{0}^{\infty}\left(\mathbf{R}^{n}\right)$ is dense in $W^{m, 2}(\Omega)$ (indeed $\partial G$ being of class $C$ is enough for this-see [10, Remark 5(a)]) and furthermore $G$ has the uniform $C^{m}$-regularity property defined by Adams in [1] (see [10, Theorem 4.2]). Consequently, on referring to [1, Theorem 7.53], the trace operators $\gamma_{j}$ :

$$
\gamma_{j} u=\left(\frac{\partial}{\partial \nu}\right)^{j} u \uparrow_{\partial G}, \quad j=0,1, \ldots, m-1,
$$

where $\nu$ is the outward normal to $\partial G$, are defined on $W^{m, 2}(G)$ and $\gamma^{j}$ maps $W^{m, 2}(G)$ continuously onto the fractional Sobolev space $W^{m-j-1 / 2,2}(\partial G)$; see [1, \$7.51] and the references therein for a definition of the latter space.

From the Embedding Theroem (see Adams [1]), $W^{m-j-1 / 2,2}(\partial G)$ is continuously embedded in $L^{p_{j}}(\partial G)$ where

$$
p_{j} \begin{cases}=2(n-1) /[n-2(m-j)] & \text { if } m-j<n / 2, \\ \in[2, \infty) & \text { if } m-j=n / 2, \\ =\infty & \text { if } m-j<n / 2 .\end{cases}
$$

Thus $\gamma_{j}$ is a continuous map of $W^{m, 2}(G)$ into $L^{p_{j}}(\partial G)$ and

$$
\left\|\gamma_{j} u\right\|_{L^{p_{j}(\partial G)}} \leqslant K\|u\|_{m, G}, \quad u \in W^{m, 2}(G), j=0,1, \ldots, m-1,
$$

for some constant $K$. Moreover, $W^{m-j-1 / 2,2}(\partial G)$ is compactly embedded in $L^{2}(\partial G)$; hence $\gamma_{j}: W^{m, 2}(G) \rightarrow L^{2}(\partial G)$ is compact and on applying [17, p. 137, Lemme 4.1], given $\varepsilon>0$ there exists a positive constant $K(\varepsilon)$ such that

$$
\left\|\gamma_{j} u\right\|_{\partial G}^{2} \leqslant \varepsilon\|u\|_{m, G}^{2}+K(\varepsilon)\|u\|_{G}^{2}, \quad u \in W^{m, 2}(G), j=0,1, \ldots, m-1 .
$$

We need to remark on three further results before proceeding. First, to say that $\partial G$ is of class $C$ is equivalent to saying that $G$ has the segment property (see [10, Definition 3.2 and Theorem 3.3]). Thus, from [2, Theorem 3.8] if $\partial G$ is of class $C$ the 
natural embedding map $W^{m, 2}(G) \rightarrow L^{2}(G)$ is compact. Secondly, from [10, Theorem 4.3(B)], if $\partial G$ is of class $C^{1}$ it is minimally smooth in the sense of Stein [32, p. 189] and hence, from [32, Theorem 5, p. 181] there exists a linear extension operator $E$ which maps $W^{k, 2}(G)$ continuously into $W^{k, 2}\left(\mathbf{R}^{n}\right)$ for all $k \in \mathbf{N}$. Thus, if $u \in$ $W^{k, 2}(G), E u \in W^{k, 2}\left(\mathbf{R}^{n}\right)$ and there exists a positive constant $K$ such that

$$
\|u\|_{k, \Omega} \leqslant\|E u\|_{k, \mathbf{R}^{n}} \leqslant K\|u\|_{k, \Omega} .
$$

If $\phi \in C_{0}^{\infty}\left(\mathbf{R}^{n}\right)$ we know that (see [11, Theorem 9.3])

$$
\left\|\nabla^{j} \boldsymbol{\phi}\right\|_{\mathbf{R}^{n}} \leqslant K\left\|\nabla^{m} \phi\right\|_{\mathbf{R}^{n}}^{j / m}\|\phi\|_{\mathbf{R}^{n}}^{1-j / m}, \quad j=0,1, \ldots, m-1,
$$

where

$$
\left\|\nabla^{j} \phi\right\|_{\mathbf{R}^{n}}^{2}=\sum_{|\alpha|=j} \int_{\mathbf{R}^{n}}\left|D^{\alpha} \phi\right|^{2}
$$

and $K$ is a positive constant depending only on $n, m$, and $j$. From (2.5) and (2.6) it follows that if $\partial G$ is of class $C^{1}$ then for $u \in W^{m, 2}(G)$,

$$
\left\|\nabla^{j} u\right\|_{G} \leqslant K\|u\|_{m, G}^{j / m}\|u\|_{G}^{1-j / m}, \quad j=0,1, \ldots, m-1 .
$$

On using Young's inequality we get for any $\delta>0$

$$
\begin{aligned}
\left\|\nabla^{j} u\right\|_{G}^{2} & \leqslant K\left\{\delta^{2 m-2 j}\|u\|_{m, G}^{2}\right\}^{j / m}\left\{\delta^{-2 j}\|u\|_{G}^{2}\right\}^{1-j / m} \\
& \leqslant K\left\{\left(\frac{j}{m}\right) \delta^{2 m-2 j}\|u\|_{m, G}^{2}+\left(\frac{m-j}{m}\right) \delta^{-2 j}\|u\|_{G}^{2}\right\} .
\end{aligned}
$$

Thus, for any $\varepsilon>0$ there exists a positive constant $K(\varepsilon)$ such that

$$
\left\|\nabla^{j} u\right\|_{G}^{2} \leqslant \varepsilon\left\|\nabla^{m}\right\|_{G}^{2}+K(\varepsilon)\|u\|_{G}^{2}, \quad j=0,1, \ldots, m-1 .
$$

Finally, from the Embedding Theorem $W^{m-j, 2}(G)$ is continuously embedded in $L^{q_{j}}(G)$ where

$$
q_{j} \begin{cases}=2 n /[n-2(m-j)] & \text { if } m-j<n / 2, \\ \in[2, \infty) & \text { if } m-j=n / 2, \\ =\infty & \text { if } m-j>n / 2,\end{cases}
$$

and hence

$$
\|u\|_{L^{q_{j}(G)}} \leqslant K\|u\|_{m-j, G}, \quad u \in W^{m-j, 2}(G) .
$$

The above remarks serve to motivate the following smoothness assumptions we make on $\partial \Omega \backslash S$. Let $\Omega_{k}, k=1,2, \ldots$, be bounded domains in $\mathbf{R}^{n}$ which satisfy

(i) $\Omega_{k} \Subset \Omega_{k+1}$;

(ii) $\bar{\Omega} \backslash S=\bigcup_{k=1}^{\infty}\left(\bar{\Omega} \cap \Omega_{k}\right)$; and

(iii) there is a $k_{0} \in \mathbf{N}$ such that for all $k \geqslant k_{0}$,

$$
\bar{\Omega} \backslash \Omega_{k} \subset \bar{\Omega} \cap\left(N_{S} \cup N_{\infty}\right),
$$

where $N_{S}$ is the neighborhood of the finite singularities, $S \backslash\{\infty\}$, mentioned above, and $N_{\infty}=\{x:|x|>K\}$ for some $K$. Our smoothness assumptions on $\partial \Omega \backslash S$ are the following. 
(A) For each $k \in \mathbf{N}$

(1) the trace operators $\gamma_{j}: \gamma_{j} u=(\partial / \partial v)^{j} u \uparrow_{\partial\left(\Omega \cap \Omega_{k}\right)}, j=0,1, \ldots, m-1$, are defined on $W^{m, 2}\left(\Omega \cap \Omega_{k}\right)$ and $\gamma_{j}$ maps $W^{m, 2}\left(\Omega \cap \Omega_{k}\right)$ continuously into $L^{p_{j}}\left(\partial\left[\Omega \cap \Omega_{k}\right]\right)$ where $p_{j}$ satisfies $(2.2)$;

(2) the embedding $W^{m, 2}\left(\Omega \cap \Omega_{k}\right) \rightarrow L^{2}\left(\Omega \cap \Omega_{k}\right)$ is compact, and

$$
W^{m-j, 2}\left(\Omega \cap \Omega_{k}\right) \rightarrow L^{q_{k}}\left(\Omega \cap \Omega_{k}\right)
$$

is continuous, where $q_{j}$ satisfies (2.10);

(3) given any $\varepsilon>0$ there exists a positive constant $K(\varepsilon)$ such that for $j=$ $0,1, \ldots, m-1$,

$$
\left\|\nabla^{j} u\right\|_{\Omega \cap \Omega_{k}}^{2} \leqslant \varepsilon\left\|\nabla^{m} u\right\|_{\Omega \cap \Omega_{k}}^{2}+K(\varepsilon)\|u\|_{\Omega \cap \Omega_{k}}^{2}
$$

and

$$
\left\|\gamma_{j} u\right\|_{\partial\left(\Omega \cap \Omega_{k}\right)}^{2} \leqslant \varepsilon\left\|\nabla^{m} u\right\|_{\Omega \cap \Omega_{k}}^{2}+K(\varepsilon)\|u\|_{\Omega \cap \Omega_{k}}^{2}
$$

for $u \in W^{m, 2}\left(\Omega \cap \Omega_{k}\right)$.

In view of the preceding remarks (A) is satisfied if $\partial\left(\Omega \cap \Omega_{k}\right)$ is of class $C^{m}$ for each $k \in \mathbf{N}$. Also note that these assumptions relate to the smoothness of $\partial \Omega \backslash S$ since the boundaries of the $\Omega_{k}$ may be chosen to be as smooth as we please.

3. The operator $\tilde{T}$. Define the differential expression

$$
\tau:=w^{-1} \sum_{j=0}^{m} \sum_{|\alpha|=|\beta|=j}(-1)^{j} D^{\alpha} a_{\alpha \beta} D^{\beta} .
$$

Let $A_{j}(x)$ denote the $n_{j}$ by $n_{j}$ matrix $\left(a_{\alpha \beta}(x)\right)$ where $n_{j}$ is the number of distinct $n$-tuples of order $j$, i.e., $|\alpha|=j$. The $n_{j}$-dimensional vector $\left(D^{\alpha} u\right)_{|\alpha|=j}$ will be denoted by $\nabla^{j} u$. For an $n_{j}$-dimensional, complex vector-valued function $\Phi=\left(\phi_{\alpha}\right)$ define

Now, we can rewrite $\tau u$ as

$$
\operatorname{div}_{j} \Phi=\sum_{|\alpha|=j} D^{\alpha} \phi_{\alpha}
$$

$$
\tau u=w^{-1} \sum_{j=0}^{m}(-1)^{j} \operatorname{div}_{j}\left(A_{j} \nabla^{j} u\right) .
$$

Let $L_{w}^{2}(\Omega)$ be the weighted space with norm

$$
\|u\|_{L_{w}^{2}(\Omega)}=\left[\int_{\Omega} w|u|^{2} d x\right]^{1 / 2} .
$$

With sufficient smoothness conditions on the coefficients of $\tau$, an operator $T$ : $\mathscr{D}(T) \rightarrow L_{w}^{2}(\Omega)$ can be defined by $T u=\tau u$ for $u \in \mathscr{D}(T) \subset L_{w}^{2}(\Omega)$ and

$$
\begin{aligned}
\mathscr{D}(T)=\{u: u= & \phi \uparrow_{\Omega}, \phi \in C_{0}^{\infty}\left(\mathbf{R}^{n} \backslash \Gamma_{S}\right), \tau u \in L_{w}^{2}(\Omega) \text { and } \\
& \mathscr{N}_{2 m-1-j}(s, D) \phi(s)+\sigma_{j}(s)\left(\frac{\partial}{\partial \nu}\right)^{j} \phi(s)=0 \\
& \text { for } \left.s \in \Gamma_{R} \text { and } j=0,1, \ldots, m-1\right\} .
\end{aligned}
$$

In (3.3), the $\sigma_{j}$ are given functions and the $\mathscr{N}_{k}(s, D)$ are linear differential operators of order $k$ on $\Gamma_{R}, \Gamma_{R}$ being noncharacteristic for each $\mathscr{N}_{k}(s, D)$-see [2, Theorem 10.2]. 
Using such an operator $T$, a sesquilinear form can be defined by

$$
t[u, v]=(T u, v), \quad \mathscr{D}(t)=\mathscr{D}(T) .
$$

If $T$ is sectorial, then the form $t$ is closable (Theorem 1.27, p. 318 of [13]). By the First Representation Theorem [13, p. 322], there is associated with the closure $\tilde{t}$ of $t$, a unique operator, the $m$-sectorial Friedrichs extension $\tilde{T}$ of $T$ for which $\mathscr{D}(\tilde{T}) \subset$ $\mathscr{D}(\tilde{t})$ and $\tilde{t}[u, v]=(\tilde{T} u, v)$ for every $u \in \mathscr{D}(\tilde{T})$ and $v \in \mathscr{D}(\tilde{t})$. In the special case that $T$ is symmetric and bounded below, $t$ will be closable and $\tilde{T}$ will be selfadjoint.

In this section we will begin by assuming that we are given a form

$$
t[u, v]=\sum_{j=0}^{m} \int_{\Omega}\left\langle A_{j} \nabla^{j} u, \nabla^{j} v\right\rangle d x+\sum_{j=0}^{m-1} \int_{\Gamma_{R}} \sigma_{j}\left(\frac{\partial}{\partial \nu}\right)^{j} u\left(\frac{\partial}{\partial \nu}\right)^{j} \bar{v} d s
$$

with domain

$$
\mathscr{D}(t)=\left\{u: u=\phi \prod_{\Omega}, \phi \in C_{0}^{\infty}\left(\mathbf{R}^{n} \backslash \Gamma_{S}\right)\right\} .
$$

The matrices $A_{j}, j=0,1, \ldots, m$, are assumed to be Hermitian and each $\sigma_{j}$ is real-valued. Consequently, $t$ is symmetric as well as being densely defined. Here, $\langle\cdot, \cdot\rangle$ denotes the $l^{2}\left(n_{j}\right)$ inner product, the dependence on $j$ being clear and hence suppressed. We allow for the fact that $t$ might be associated with an operator such as $T$ above, but this is not required. We will need for $t$ to be closable. The results of subsequent sections will be related to the spectrum of the operator $\tilde{T}$ associated with $\tilde{t}$ as guaranteed by the First Representation Theorem. The fact that $t$ is closable may either arise from its association with a densely defined, semibounded, symmetric operator $T$ as above or it may arise from the inherent properties of the form itself. We illustrate below in Lemma 2 how the latter may occur.

The following will comprise our basic assumptions on the coefficients in (3.5). The smallest and largest eigenvalue of each $A_{j}(x)$ is denoted by $\mu_{j}(x)$ and $\lambda_{j}(x)$, respectively. The norm of each $A_{j}$ as a symmetric operator in $l^{2}\left(n_{j}\right)$ is $\left|A_{j}(x)\right|:=\max \left(\left|\mu_{j}(x)\right|,\left|\lambda_{j}(x)\right|\right)$.

Assume that

(B)(1) $\mu_{m}>0$ a.e. on $\Omega, \mu_{m}^{-1} \in L^{\infty}\left(\Omega \cap \Omega_{k}\right)$ for $k \in \mathbf{N}$,

(2) $A_{0}$ is real-valued, for $k \in \mathbf{N} A_{0} \in L^{\alpha_{0}}\left(\Omega \cap \Omega_{k}\right)$ and $\left|A_{j}\right| \in L^{\alpha_{j}}\left(\Omega \cap \Omega_{k}\right)$, $j=1,2, \ldots, m-1$, where

$$
\alpha_{j} \begin{cases}=n / 2(m-j) & \text { if } m-j<n / 2, \\ \in(1, \infty] & \text { if } m-j=n / 2, \\ =1 & \text { if } m-j>n / 2 .\end{cases}
$$

(C)(1) For $j=0,1, \ldots, m-1$, assume that $\sigma_{j} \in L^{\beta_{j}}\left(\Gamma_{R}\right)$ where

$$
\beta_{j} \begin{cases}=(n-1) / 2(m-j)-1 & \text { if } m-j<n / 2, \\ \in(1, \infty] & \text { if } m-j=n / 2, \\ =1 & \text { if } m-j>n / 2,\end{cases}
$$

(2) and $\sigma_{j} \geqslant 0, j=0,1, \ldots, m-1$, in $\Gamma_{R} \backslash \bar{\Omega}_{k}$ for $k \geqslant k_{0}$ defined in (2.12).

(D) For a.e. $x \in \Omega$ assume that $w(x)>0$ and let $w^{-1} \in L^{\infty}\left(\Omega \cap \Omega_{k}\right), k \in \mathbf{N}$. 
LEMMA 1. Assume (A)-(D). For each $k \in \mathbf{N}$ and arbitrarily small positive numbers $\varepsilon_{1}, \varepsilon_{2}$, and $\varepsilon_{3}$, there are constants $K\left(\varepsilon_{1}\right), K\left(\varepsilon_{2}\right)$, and $K\left(\varepsilon_{3}\right)$ such that for all $u \in \mathscr{D}(t)$

$$
\begin{aligned}
\int_{\Omega \cap \Omega_{k}}\left|A_{j}(x)\right|\left|\nabla^{i} u\right|^{2} d x & \leqslant \varepsilon_{1}\left\|\nabla^{m} u\right\|_{\Omega \cap \Omega_{k}}^{2}+K\left(\varepsilon_{1}\right)\|u\|_{\Omega \cap \Omega_{k}}^{2} \\
& \leqslant \varepsilon_{2} \int_{\Omega \cap \Omega_{k}} \mu_{m}\left|\nabla^{m} u\right|^{2} d x+K\left(\varepsilon_{2}\right) \int_{\Omega \cap \Omega_{k}} w(x)|u(x)|^{2} d x
\end{aligned}
$$

for $0 \leqslant i \leqslant j \leqslant m-1$, and

$$
\begin{aligned}
& \int_{\Gamma_{R} \cap \bar{\Omega}_{k}}\left|\sigma_{j}\right|\left|\left(\frac{\partial}{\partial \nu}\right)^{j} u\right|^{2} d s \\
& \quad \leqslant \varepsilon_{3} \int_{\Omega \cap \Omega_{k}} \mu_{m}\left|\nabla^{m} u\right|^{2} d x+K\left(\varepsilon_{3}\right) \int_{\Omega \cap \Omega_{k}}|u(x)|^{2} d x \\
& \quad \leqslant \varepsilon_{3} \int_{\Omega \cap \Omega_{k}} \mu_{m}\left|\nabla^{m} u\right|^{2} d x+K\left(\varepsilon_{3}\right) \int_{\Omega \cap \Omega_{k}} w(x)|u(x)|^{2} d x .
\end{aligned}
$$

Proof. The exponents $\alpha_{j}$ in (3.7) satisfy $1 / 2 \alpha_{j}+1 / q_{j}=1 / 2$, for $q_{j}$ given by (2.10). Therefore, by (2.11) and Hölder's inequality $\left|A_{j}\right|^{1 / 2}\left|\nabla^{j} u\right| \in L^{2}\left(\Omega \cap \Omega_{k}\right)$ for $u \in \mathscr{D}(t)$. Given any $\varepsilon^{\prime}>0$ we can write $\left|A_{j}\right|=f_{j, 1}+f_{j, 2}$ where for each $k \in \mathbf{N}$ and some $K\left(\varepsilon^{\prime}\right)>0$ depending on $\varepsilon^{\prime}$ and $\Omega \cap \Omega_{k}$,

$$
\left\|f_{j, 1}\right\|_{L^{\alpha_{j}\left(\Omega \cap \Omega_{k}\right)}}<\varepsilon^{\prime}, \quad\left|f_{j, 2}\right|<K\left(\varepsilon^{\prime}\right) \quad \text { on } \Omega \cap \Omega_{k} .
$$

Hence, from (B)(2), for $u \in \mathscr{D}(t)$ and $0 \leqslant i \leqslant j \leqslant m-1$,

$$
\begin{aligned}
\int_{\Omega \cap \Omega_{k}}\left|A_{j}\right|\left|\nabla^{i} u\right|^{2} d x & \leqslant\left\|f_{j, 1}\right\|_{L^{\alpha_{j}\left(\Omega \cap \Omega_{k}\right)}}\left\|\nabla^{i} u\right\|_{L^{q_{j}\left(\Omega \cap \Omega_{k}\right)}}^{2}+K\left(\varepsilon^{\prime}\right)\left\|\nabla^{i} u\right\|_{\Omega \cap \Omega_{k}}^{2} \\
& \leqslant \varepsilon^{\prime} K\|u\|_{m, \Omega \cap \Omega_{k}}^{2}+\varepsilon^{\prime}\left\|\nabla^{m} u\right\|_{\Omega \cap \Omega_{k}}^{2}+K\left(\varepsilon^{\prime}\right)\|u\|_{\Omega \cap \Omega_{k}}^{2} \\
& \leqslant \varepsilon^{\prime \prime}\left\|\nabla^{m} u\right\|_{\Omega \cap \Omega_{k}}^{2}+K\left(\varepsilon^{\prime \prime}\right)\|u\|_{\Omega \cap \Omega_{k}}^{2} \\
& \leqslant \varepsilon \int_{\Omega \cap \Omega_{k}} \mu_{m}\left|\nabla^{m} u\right|^{2} d x+K(\varepsilon) \int_{\Omega \cap \Omega_{k}}|u|^{2} w d x
\end{aligned}
$$

by (2.11), (2.13), (B)(1), (D), and the fact that $q_{i} \geqslant q_{j}$.

Similarly, the exponents $\beta_{j}$ in (C) satisfy $1 / \beta_{j}+2 / p_{j}=1$, for $p_{j}$ given by (2.2). Hence, for $u \in \mathscr{D}(t)$ as above we have (3.10), on using (2.3) and (2.4) and extending $\sigma_{j}$ as zero on $\partial \Omega_{k} \backslash \partial \Omega$.

We define $f^{-}(x)=\min \{f(x), 0\}$ and $f^{+}(x)=\max \{f(x), 0\}$.

Lemma 2. Assume (A)-(D), and that $A_{j} \geqslant 0, j=1,2, \ldots, m-1$, a.e. on $\Omega \backslash \Omega_{k}$ for some $k_{1} \in \mathbf{N}$ and all $k \geqslant k_{1}$. If

$$
\lim _{k \rightarrow \infty} \inf _{x \in \Omega \backslash \Omega_{k}} \frac{A_{0}(x)}{w(x)}=: a_{0}>-\infty,
$$

then $t$ is bounded below and closable in $L_{w}^{2}(\Omega)$. 
Proof. By the Principal Axes Theorem each Hermitian matrix $A_{j}, j=0,1, \ldots, m$, is simultaneously similar to and conjunctive to a real diagonal matrix $D$ having eigenvalues of $A_{j}$ as the diagonal elements. Consequently, we can write $A_{j}=A_{j}^{+}+$ $A_{j}^{-}$where $A_{j}^{+} \geqslant 0, A_{j}^{-} \leqslant 0$ and each matrix is Hermitian. (Of course, $A_{m}=A_{m}^{+}>0$.)

Let $\varepsilon \in(0,1)$ and $u \in \mathscr{D}(t)$. Then for $k$ sufficiently large

$$
\begin{aligned}
t[u] \geqslant & \sum_{j=0}^{m} \int_{\Omega}\left\langle A_{j}^{+} \nabla^{j} u, \nabla^{j} u\right\rangle d x-\sum_{j=0}^{m-1} \int_{\Omega \cap \Omega_{k}}\left|A_{j}\right|\left|\nabla^{j} u\right|^{2} d x \\
& +\left(a_{0}^{-}-\varepsilon\right) \int_{\Omega \backslash \Omega_{k}}|u|^{2} w d x+\sum_{j=0}^{m-1} \int_{\Gamma_{R}} \sigma_{j}^{+}\left|\left(\frac{\partial}{\partial \nu}\right)^{j} u\right|^{2} d s \\
& -\sum_{j=0}^{m-1} \int_{\Gamma_{R} \cap \bar{\Omega}_{k}}\left|\sigma_{j}\right|\left|\left(\frac{\partial}{\partial \nu}\right)^{j} u\right|^{2} d s \\
\geqslant & \sum_{j=0}^{m} \int_{\Omega}\left\langle A_{j}^{+} \nabla^{j} u, \nabla^{j} u\right\rangle d x \\
& -\sum_{j=0}^{m-1}\left\{\frac{\varepsilon}{m} \int_{\Omega \cap \Omega_{k}} \mu_{m}\left|\nabla^{m} u\right|^{2} d x+K(\varepsilon) \int_{\Omega \cap \Omega_{k}}|u|^{2} w d x\right\} \\
& +\left(a_{0}^{-}-\varepsilon\right) \int_{\Omega \backslash \Omega_{k}}|u|^{2} w d x+\sum_{j=0}^{m-1} \int_{\Gamma_{R}} \sigma_{j}^{+}\left|\left(\frac{\partial}{\partial \nu}\right)^{j} u\right|^{2} d s \\
\geqslant & (1-\varepsilon) \sum_{j=0}^{m} \int_{\Omega}\left\langle A_{j}^{+} \nabla^{j} u, \nabla^{j} u\right\rangle d x+\left(a_{0}^{-}-K(\varepsilon)\right)\|u\|_{L_{w}^{2}(\Omega)} \\
& +\sum_{j=0}^{m-1} \int_{\Gamma_{R}} \sigma_{j}^{+}\left|\left(\frac{\partial}{\partial \nu}\right)^{j} u\right|^{2} d s
\end{aligned}
$$

by Lemma 1. From (3.12) we can conclude that $t$ is bounded below by $a_{0}^{-}-K(\varepsilon)$ in $L_{w}^{2}(\Omega)$.

It remains to prove that $t$ is closable. In order to do this we need to show that if $\left\{u_{l}\right\} \in \mathscr{D}(t)$ is $t$-convergent to 0 , i.e., $u_{l} \rightarrow 0$ in $L_{w}^{2}(\Omega)$ and $t\left[u_{l}-u_{r}\right] \rightarrow 0$ as $l, r \rightarrow \infty$, then $t\left[u_{l}\right] \rightarrow 0$ as $l \rightarrow \infty$.

First, we will show that

$$
\int_{\Omega}\left\langle A_{j}^{+} \nabla^{j} u_{l}, \nabla^{j} u_{l}\right\rangle d x \rightarrow 0 \quad \text { as } l \rightarrow \infty
$$

for $j=0,1, \ldots, m$. Let $A_{j}^{+}(x)$ have eigenvalues $\delta_{i j}(x)$ and orthonormal eigenvectors $\hat{\phi}_{i j}(x)$. Then

$$
\left\langle A_{j}^{+} \nabla^{j} u, \nabla^{j} u\right\rangle=\sum_{i} \delta_{i j}\left|\left\langle\nabla^{j} u, \hat{\phi}_{i j}\right\rangle\right|^{2} .
$$

It follows from (3.12) and the fact that $\left\{u_{l}\right\}$ is $t$-convergent to 0 that

$$
\sum_{i} \int_{\Omega} \delta_{i j}\left|\left\langle\nabla^{j} u_{l}, \hat{\phi}_{i j}\right\rangle-\left\langle\nabla^{j} u_{r}, \hat{\phi}_{i j}\right\rangle\right|^{2} d x \rightarrow 0
$$


as $l, r \rightarrow \infty$. Since $L^{2}\left(\Omega ; \delta_{i j} d x\right)$ is complete, there exists $v_{i j} \in L^{2}\left(\Omega ; \delta_{i j} d x\right)$ such that for each $i$

$$
\int_{\Omega} \delta_{i j}\left|\left\langle\nabla^{j} u_{l}, \hat{\phi}_{i j}\right\rangle-v_{i j}\right|^{2} d x \rightarrow 0
$$

as $l \rightarrow \infty$. Thus, for $\hat{v}_{j}(x):=\sum_{i} v_{i j}(x) \hat{\phi}_{i j}(x)$

$$
\sum_{i} \int_{\Omega} \delta_{i j}\left|\left\langle\nabla^{j} u_{l}-\hat{v}_{j}, \hat{\boldsymbol{\phi}}_{i j}\right\rangle\right|^{2} d x \rightarrow 0
$$

as $l \rightarrow \infty$. Hence, by (B)(1)

$$
\sum_{i} \int_{\Omega \cap \Omega_{k}}\left|\left\langle\nabla^{m} u_{l}-\hat{v}_{m}, \hat{\phi}_{i m}\right\rangle\right|^{2} d x \rightarrow 0
$$

as $l \rightarrow \infty$, for each $k$. We will now show that $\hat{v}_{m}=0$ a.e. in $\Omega$ from which (3.13) will follow for $j=m$. By (D) and the fact that $u_{l} \rightarrow 0$ in $L_{w}^{2}(\Omega)$, it follows that

$$
\int_{\Omega} u_{l} D_{j}^{\alpha} d x \rightarrow 0 \text { as } l \rightarrow \infty,
$$

for any $\psi \in C_{0}^{\infty}(\Omega)$, or equivalently that

$$
\int_{\Omega} D^{\alpha} u_{l} \psi d x \rightarrow 0 \text { as } l \rightarrow \infty,
$$

for all $\alpha$ with $0 \leqslant|\alpha| \leqslant m$. Thus, if $\hat{\psi}_{j}=\left(\psi_{1}, \ldots, \psi_{n_{j}}\right)$ with components being $C_{0}^{\infty}(\Omega)$-functions, then

$$
\int_{\Omega}\left\langle\nabla^{m} u_{l}, \hat{\psi}_{m}\right\rangle d x \rightarrow 0 \text { as } l \rightarrow \infty .
$$

By (2.12), supp $\hat{\psi}_{m} \subseteq \Omega \cap \Omega_{k}$ for $k$ sufficiently large. Hence, by (3.15) and the Cauchy-Schwarz inequality,

$$
\begin{aligned}
\int_{\Omega}\left\langle\nabla^{m} u_{l}, \hat{\psi}_{m}\right\rangle d x & =\sum_{i} \int_{\Omega}\left\langle\nabla^{m} u_{l}, \hat{\boldsymbol{\phi}}_{i m}\right\rangle\left\langle\overline{\hat{\psi}_{m}, \hat{\boldsymbol{\phi}}_{i m}}\right\rangle d x \\
& \rightarrow \sum_{i} \int_{\Omega}\left\langle\hat{v}_{m}, \hat{\boldsymbol{\phi}}_{i m}\right\rangle\left\langle\overline{\hat{\psi}_{i m}, \hat{\boldsymbol{\phi}}_{m}}\right\rangle d x \\
& =\int_{\Omega}\left\langle\hat{v}_{m}, \hat{\psi}_{m}\right\rangle d x .
\end{aligned}
$$

Thus, on using (3.16) $\int_{\Omega}\left\langle\hat{v}_{m}, \hat{\psi}_{m}\right\rangle d x=0$ for an arbitrary vector-valued function $\hat{\psi}_{m}$ with components in $C_{0}^{\infty}(\Omega)$. Hence, $v_{m}=0$ a.e. in $\Omega$ and (3.13) is proved for $j=m$. By (3.9), we can now conclude that the limit in (3.13) holds for $j=0,1, \ldots, m$, when $\Omega$ is replaced by $\Omega \cap \Omega_{k}$. Since supp $\hat{\psi}_{j} \subseteq \Omega \cap \Omega_{k}$ for $k$ sufficiently large and $A_{j}^{+} \geqslant 0$, then by the Cauchy-Schwarz inequality

$$
\begin{aligned}
\int_{\Omega}\left\langle A_{j}^{+} \nabla^{j} u_{l}, \hat{\psi}_{j}\right\rangle d x & =\int_{\Omega \cap \Omega_{k}}\left\langle A_{j}^{+} \nabla^{j} u_{l}, \hat{\psi}_{j}\right\rangle d x \\
& \leqslant\left[\int_{\Omega \cap \Omega_{k}}\left\langle A_{j}^{+} \nabla^{j} u_{l}, \nabla^{j} u_{l}\right\rangle d x\right]^{1 / 2}\left[\int_{\Omega \cap \Omega_{k}}\left\langle A_{j}^{+} \hat{\psi}_{j}, \hat{\psi}_{j}\right\rangle d x\right]^{1 / 2} \\
& \rightarrow 0
\end{aligned}
$$


as $l \rightarrow \infty$. However, by (3.14)

$$
\begin{aligned}
\int_{\Omega}\left\langle A_{j}^{+} \nabla^{j} u_{l}, \hat{\psi}_{j}\right\rangle d x & =\sum_{i} \int_{\Omega} \delta_{i, j}\left\langle\nabla^{j} u_{l}, \hat{\phi}_{i j}\right\rangle\left\langle\overline{\hat{\psi}_{j}, \hat{\phi}_{i j}}\right\rangle d x \\
& \rightarrow \sum_{i} \int_{\Omega} \delta_{i, j}\left\langle\hat{v}_{j}, \hat{\phi}_{i, j}\right\rangle\left\langle\overline{\hat{\psi}_{j}, \hat{\phi}_{i j}}\right\rangle d x \\
& =\int_{\Omega}\left\langle A_{j}^{+} \hat{v}_{j}, \hat{\psi}_{j}\right\rangle d x .
\end{aligned}
$$

As above, we can conclude that $A_{j}^{+} \hat{v}_{j} \equiv 0$ a.e. in $\Omega$. Since in (3.14)

$$
\sum_{i} \int_{\Omega} \delta_{i, j}\left|\left\langle\nabla^{j} u_{l}-\hat{v}_{j}, \hat{\phi}_{i j}\right\rangle\right|^{2} d x=\int_{\Omega}\left\langle A_{j}^{+}\left(\nabla^{j} u_{l}-\hat{v}_{j}\right), \nabla^{j} u_{l}-\hat{v}_{j}\right\rangle d x
$$

then by (3.14), (3.13) is proved.

Since for any $u \in \mathscr{D}(t)$

$$
t[u] \leqslant \sum_{j=0}^{m} \int_{\Omega}\left\langle A_{j}^{+} \nabla^{j} u, \nabla^{j} u\right\rangle d x+\sum_{j=0}^{m-1} \int_{\Gamma_{R}} \sigma_{j}^{+}\left|\left(\frac{\partial}{\partial \nu}\right)^{j} u\right|^{2} d s,
$$

the proof will be complete if we can show that

$$
\sum_{j=0}^{m-1} \int_{\Gamma_{R}} \sigma_{j}^{+}\left|\left(\frac{\partial}{\partial \nu}\right)^{j} u_{l}\right|^{2} d s \rightarrow 0, \quad \text { as } l \rightarrow \infty .
$$

Since $t\left[u_{l}-u_{r}\right] \rightarrow 0$ as $l, r \rightarrow \infty$, by (3.12), there exists $v_{j}$ for each $j=$ $0,1, \ldots, m-1$, such that

$$
\int_{\Gamma_{R}} \sigma_{j}^{+}\left|\left(\frac{\partial}{\partial \nu}\right)^{j} u_{l}-v_{j}\right|^{2} d s \rightarrow 0 \quad \text { as } l \rightarrow \infty .
$$

By (3.10) and (3.13) we can conclude that $v_{j}=0$ a.e. on $\Gamma_{R} \cap \Omega_{k}$ for each $k$. However, $\Gamma_{R}=\bigcup_{k}\left(\Gamma_{R} \cap \Omega_{k}\right)$. Therefore, $v_{j}=0$ a.e. on $\Gamma_{R}$ and (3.17) follows. The proof of Lemma 2 is thus complete.

4. Lower bound of the essential spectrum of $\tilde{T}$. The essential spectrum $\sigma_{e}(\tilde{T})$ of the selfadjoint operator $\tilde{T}$ is defined to be the complement in the spectrum $\sigma(\tilde{T})$ of $\tilde{T}$ of the set of isolated eigenvalues of finite multiplicity. Equivalently, it is the set of points $\lambda \in \mathbf{C}$ for which there exist singular sequences; $\left\{u_{l}\right\}$ is a singular sequence for $\lambda$ if $\left\|u_{l}\right\|_{L_{w}^{2}(\Omega)}=1, u_{l} \rightarrow 0$ in $L_{w}^{2}(\Omega)$, and $(\tilde{T}-\lambda I) u_{l} \rightarrow 0$ in $L_{w}^{2}(\Omega)$. Our immediate concern is with the number

$$
l_{e}:=\inf \left\{\lambda: \lambda \in \sigma_{e}(\tilde{T})\right\} .
$$

In this section we assume the hypothesis of Lemma 2. From Lemma 2 we may further assume, without loss of generality, that $t[u] \geqslant\|u\|_{L_{w}^{2}(\Omega)}^{2}$ on $\mathscr{D}(t)$ (and hence $\tilde{T} \geqslant 1$ ) and that in (3.11) $a_{0}>0$. This property can be effected by the addition of a sufficiently large positive constant to $\tilde{T}$ which merely translates $\sigma_{e}(\tilde{T}) . \tilde{T}$ therefore has a square root $\tilde{T}^{1 / 2}$ and from the Second Representation Theorem [13, Theorem VI-2.23] we have that $\mathscr{D}(\tilde{t})=\mathscr{D}\left(\tilde{T}^{1 / 2}\right)$ and

$$
\tilde{t}[u, v]=\left(\tilde{T}^{1 / 2} u, T^{1 / 2} v\right)_{L_{w}^{2}(\Omega)}=:(u, v)_{\tilde{i}}
$$


Furthermore $\tilde{T} \geqslant 1$ implies that $\|\cdot\|_{i}$ is equivalent to the graph norm of $\tilde{T}^{1 / 2}$ on $\mathscr{D}(\tilde{t})$ and $\left(\mathscr{D}(\tilde{t}) ;\|\cdot\|_{i}\right)$ is a Hilbert space which is continuously embedded in $L_{w}^{2}(\Omega)$; we shall denote $\left(\mathscr{D}(\tilde{t}) ;\|\cdot\|_{\tilde{i}}\right)$ by $H(\tilde{t}) . \mathscr{D}(t)$ is a dense subspace of $H(\tilde{t})$.

To determine $l_{e}$ we invoke Theorem 3.3 in [8] with the following choices for the operators and spaces. The closable operator $T$ in [8] is taken to be $\tilde{T}^{1 / 2} \uparrow \mathscr{D}(t)$ and with $Y(\Omega)=L_{w}^{2}(\Omega)$, the closure of $T$ is $\tilde{T}^{1 / 2} . X$ is now the above Hilbert space $H(\tilde{t}), E=\mathscr{D}(t)$, and $Q$ the embedding map $H(\tilde{t}) \rightarrow L_{w}^{2}(\Omega)$. The form of $[8$, Theorem 3.3] which is ideally suited to our present needs incorporates the hypothesis in Remark 3 of [8]. This has the following form in the terminology of this paper:

(H) For each $k \in \mathbf{N}$ large enough and $\phi \in C_{0}\left(\mathbf{R}^{n} \backslash \Gamma_{S}\right)$ such that

$$
\phi(x)= \begin{cases}1, & x \in \Omega_{k}, \\ 0, & x \notin \Omega_{k+1},\end{cases}
$$

and $0 \leqslant \phi \leqslant 1$, we have

(i) $\phi v \in \mathscr{D}(t)$ for every $v \in \mathscr{D}(t)$,

(ii) if $v_{l} \in \mathscr{D}(t)$ satisfies $\left\|v_{l}\right\|_{\tilde{t}}=1$ and $v_{l} \rightarrow 0$ in $H(\tilde{t})$ then

$$
\left\|(1-\phi) v_{l}\right\|_{i}^{2} \leqslant 1+o(1) \quad \text { as } l \rightarrow \infty .
$$

If hypothesis $(\mathrm{H})$ is satisfied, the conclusion is (see [8, Remark 4]) that

$$
r_{e}^{2}\left(\tilde{T}^{-1 / 2}\right)=\lim _{k \rightarrow \infty} \sup \left\{\|u\|_{L_{w}^{2}\left(\Omega \backslash \Omega_{k}\right)}^{2}: u \in \mathscr{D}(t),\|u\|_{\tilde{i}}=1, \operatorname{supp} u \subset \Omega \backslash \Omega_{k}\right\}
$$

where $r_{e}\left(\tilde{T}^{-1 / 2}\right)=\left\{|\lambda|: \lambda \in \sigma_{e}\left(\tilde{T}^{-1 / 2}\right)\right\}$, the radius of the essential spectrum of $\tilde{T}^{-1 / 2}$. From this and the Spectral Mapping Theorem it will follow that

$$
\begin{array}{r}
l_{e}=r_{e}^{-2}\left(\tilde{T}^{-1 / 2}\right)=\lim _{k \rightarrow \infty} \inf \left\{t[u]: u \in \mathscr{D}(t),\|u\|_{L_{w}^{2}(\Omega)}=1,\right. \\
\left.\operatorname{supp} u \subset \Omega \backslash \Omega_{k}\right\}
\end{array}
$$

which is the result we seek. Note that in (4.3) the statement $u \in \mathscr{D}(t)$ and supp $u \subset \Omega \backslash \Omega_{k}$ means $u=\phi \prod_{\Omega}$ for some $\phi \in C_{0}^{\infty}\left(\mathbf{R}^{n} \backslash \Omega_{k}\right)$. It may be the case that $u$ does not vanish on $\partial \Omega \backslash \bar{\Omega}_{k}$.

THEOREM 3. Assume the hypothesis of Lemma 2 and suppose that

$$
\lambda_{m} \in L^{\infty}\left(\Omega \cap \Omega_{k}\right), \quad k \in \mathbf{N} .
$$

Then

$$
l_{e}=\lim _{k \rightarrow \infty} \inf \left\{t[u]: u \in \mathscr{D}(t),\|u\|_{L_{w}^{2}(\Omega)}=1, \operatorname{supp} u \subset \Omega \backslash \Omega_{k}\right\} .
$$

Proof. It suffices to prove that hypothesis $(\mathrm{H})$ is satisfied; (4.4) will then follow from (4.3).

First, (H)(i) is obvious. We proceed then to prove (H)(ii). Let $v \in D(t)$ and take $k$ to be large enough for $\mu_{j} \geqslant 0, \sigma_{j} \geqslant 0$, and $A_{0} / w>a_{0}-\varepsilon>0$ in $\Omega \backslash \Omega_{k}$ for $j=0,1, \ldots, m-1$. Then,

$$
\begin{aligned}
\|(1-\phi) v\|_{i}^{2}= & \sum_{j=0}^{m} \int_{\Omega}\left\langle A_{j} \nabla^{j}(1-\phi) v, \nabla^{j}(1-\phi) v\right\rangle d x \\
& +\sum_{j=0}^{m-1} \int_{\Gamma_{R}} \sigma_{j}\left|\left(\frac{\partial}{\partial \nu}\right)^{j}(1-\phi) v\right|^{2} d s .
\end{aligned}
$$


208

W. D. EVANS AND R. T. LEWIS

$$
\begin{aligned}
& \text { For } j \geqslant 1, \\
& \begin{aligned}
\left\langle A_{j} \nabla^{j}(1-\phi) v, \nabla^{j}(1-\phi) v\right\rangle= & \sum_{|\alpha|=|\beta|=j} a_{\alpha \beta} D^{\beta}[(1-\phi) v] D^{\alpha}[(1-\phi) v] \\
= & \sum_{|\alpha|=|\beta|=j} a_{\alpha \beta}\left\{(1-\phi) D^{\beta} v+\sum_{0<r \leqslant \beta}\left(\begin{array}{l}
\beta \\
r
\end{array}\right) D^{r}(1-\phi) D^{\beta-r} v\right\} \\
& \times\left\{(1-\phi) D^{\alpha} v+\sum_{0<\delta \leqslant \alpha}\left(\begin{array}{l}
\alpha \\
\delta
\end{array}\right) D^{\delta}(1-\phi) D^{\alpha-\delta} v\right\} \\
\leqslant & (1-\phi)^{2}\left\langle A_{j} \nabla^{j} v, \nabla^{j} v\right\rangle \\
& +K(\phi)\left|A_{j}\right|\left\{\left|\nabla^{j} v\right| \sum_{i=0}^{j-1}\left|\nabla^{i} v\right|+\sum_{i=0}^{j-1}\left|\nabla^{i} v\right|^{2}\right\}
\end{aligned}
\end{aligned}
$$

by Leibnitz's Theorem. Here, $K(\phi)$ has support in $\Omega_{k+1} \backslash \Omega_{k}$ and depends on the distance between $\Omega_{k}$ and $\Omega_{k+1}$. Thus, for arbitrary $\varepsilon, \varepsilon_{1}, \varepsilon_{2}, \ldots$

(4.5)

$$
\begin{aligned}
\sum_{j=0}^{m} \int_{\Omega} & \left\langle A_{j} \nabla^{j}(1-\phi) v, \nabla^{j}(1-\phi) v\right\rangle d x \\
\leqslant & \sum_{j=0}^{m} \int_{\Omega}(1-\phi)^{2}\left\langle A_{j} \nabla^{j} v, \nabla^{j} v\right\rangle d x \\
& +K(\phi) \sum_{j=1}^{m} \sum_{i=0}^{j-1} \int_{\Omega \cap \Omega_{k+1}}\left|A_{j}\right|\left|\nabla^{i} v\right|\left\{\left|\nabla^{j} v\right|+\left|\nabla^{i} v\right|\right\} d x \\
\leqslant & \sum_{j=0}^{m} \int_{\Omega}\left\langle A_{j} \nabla^{j} v, \nabla^{j} v\right\rangle d x-\sum_{j=0}^{m-1} \int_{\Omega \cap \Omega_{k}}\left\langle A_{j} \nabla^{j} v, \nabla^{j} v\right\rangle d x \\
& +K(\phi) \sum_{j=1}^{m} \sum_{i=0}^{j-1} \int_{\Omega \cap \Omega_{k+1}}\left|A_{j}\right|\left|\nabla^{i} v\right|\left\{\left|\nabla^{j} v\right|+\left|\nabla^{i} v\right|\right\} d x \\
\leqslant & \sum_{j=0}^{m} \int_{\Omega}\left\langle A_{j} \nabla^{j} v, \nabla^{j} v\right\rangle d x+\sum_{j=0}^{m-1} \int_{\Omega \cap \Omega_{k}}\left|A_{j}\right|\left|\nabla^{j} v\right|^{2} d x \\
& +K(\phi) \sum_{j=1}^{m-1} \sum_{i=0}^{j-1}\left\{\left(\int_{\Omega \cap \Omega_{k+1}}\left|A_{j}\right|\left|\nabla^{i} v\right|^{2} d x\right)^{1 / 2}\left(\int_{\Omega \cap \Omega_{k+1}}\left|A_{j}\right|\left|\nabla^{j} v\right|^{2} d x\right)^{1 / 2}\right. \\
\leqslant & \left.\sum_{j=0}^{m} \int_{\Omega}\left\langle A_{j} \nabla^{j} v, \nabla^{j} v\right\rangle+\varepsilon_{1}\left\|\mu_{m}^{1 / 2} \nabla^{m} v\right\|_{\Omega \cap \Omega_{k+1}}^{2}+K\left(\varepsilon_{1}\right)\|v\|_{\Omega \cap \Omega_{k+1}}^{2}+\int_{\Omega \cap \Omega_{k+1}}\left|A_{j}\right|\left|\nabla^{i} v\right|^{2} d x\right\} \\
& +K(\phi) \sum_{i=0}^{m-1}\left\{\left(\int_{\Omega \cap \Omega_{k+1}}\left|\nabla^{i} v\right|^{2} d x\right)^{1 / 2}\left(\left.\int_{\Omega \cap \Omega_{k+1}}\left|\nabla^{m} v\right|^{2} d x\right|^{1 / 2} d x\right\}\right.
\end{aligned}
$$


from (3.9) and (2.13). Also for $j=1, \ldots, m-1$,

$$
\begin{aligned}
\int_{\Gamma_{R}} \sigma_{j}\left|\left(\frac{\partial}{\partial \nu}\right)^{j}[(1-\phi) v]\right|^{2} d s \leqslant & \int_{\Gamma_{R}}(1-\phi)^{2} \sigma_{j}\left|\left(\frac{\partial}{\partial \nu}\right)^{j} v\right|^{2} d x \\
& +K(\phi) \sum_{i=0}^{j-1} \int_{\Gamma_{R} \cap\left(\Omega_{k+1} \backslash \Omega_{k}\right)}\left|\sigma_{j}\right|\left|\left(\frac{\partial}{\partial \nu}\right)^{i} v\right|^{2} d s .
\end{aligned}
$$

Consequently,

$$
\begin{aligned}
& \sum_{j=0}^{m-1} \int_{\Gamma_{R}} \sigma_{j}\left|\left(\frac{\partial}{\partial \nu}\right)^{j}[(1-\phi) v]\right|^{2} d s \\
& \leqslant \sum_{j=0}^{m-1} \int_{\Gamma_{R}} \sigma_{j}\left|\left(\frac{\partial}{\partial \nu}\right)^{j} v\right|^{2} d s-\sum_{j=0}^{m-1} \int_{\Gamma_{R} \cap \Omega_{k}} \sigma_{j}\left|\left(\frac{\partial}{\partial \nu}\right)^{j} v\right|^{2} d s \\
& \quad+K(\phi) \sum_{j=1}^{m-1} \sum_{i=0}^{j-1} \int_{\Gamma_{R} \cap\left(\Omega_{k+1} \backslash \Omega_{k}\right)} \sigma_{j}\left|\left(\frac{\partial}{\partial \nu}\right)^{i} v\right|^{2} d s \\
& \leqslant \sum_{j=0}^{m-1} \int_{\Gamma_{R}} \sigma_{j}\left|\left(\frac{\partial}{\partial \nu}\right)^{j} v\right|^{2} d s+\varepsilon_{2}\left\|\mu_{m}^{1 / 2} \nabla^{m} v\right\|_{\Omega \cap \Omega_{k+1}}^{2}+K\left(\varepsilon_{2}\right)\|v\|_{\Omega \cap \Omega_{k+1}}^{2}
\end{aligned}
$$

on using (3.10). Thus we have proved that

$$
\|(1-\phi) v\|_{i}^{2} \leqslant\|v\|_{i}^{2}+\varepsilon_{3}\left\|\mu_{m}^{1 / 2} \nabla^{m} v\right\|_{\Omega \cap \Omega_{k+1}}^{2}+K\left(\varepsilon_{2}\right)\|v\|_{\Omega \cap \Omega_{k+1}}^{2} .
$$

From the proof of (3.12) (with $a_{0}-\varepsilon>0$ ) and (3.10) we can conclude that

$$
\|v\|_{i}^{2}=t[v] \geqslant\left(1-\varepsilon_{4}\right) \int_{\Omega \cap \Omega_{k+1}} \mu_{m}\left|\nabla^{m} v\right|^{2} d x-K\left(\varepsilon_{4}\right) \int_{\Omega \cap \Omega_{k+1}}|v|^{2} d x
$$

and so, on substituting for $\left\|\mu_{m}^{1 / 2} \nabla^{m} v\right\|_{\Omega \cap \Omega_{k+1}}^{2}$ in (4.6),

$$
\|(1-\phi) v\|_{i}^{2} \leqslant(1+\varepsilon)\|v\|_{i}^{2}+K(\varepsilon, \phi)\|v\|_{\Omega \cap \Omega_{k+1}}^{2} .
$$

It follows from equations (4.7) and (2.9) that $H(\tilde{t})$ is continuously embedded in $W^{m, 2}\left(\Omega \cap \Omega_{k+1}\right)$ and hence compactly embedded in $L^{2}\left(\Omega \cap \Omega_{k+1}\right)$ by (A)(2). Thus if $\left\{v_{l}\right\}$ satisfies the hypothesis of $(\mathrm{H})\left(\mathrm{ii)}\right.$ we have $\left\|v_{l}\right\|_{\Omega \cap \Omega_{k+1}}=o(1)$ as $l \rightarrow \infty$. Hence, from (4.8)

$$
\left\|(1-\phi) v_{l}\right\|_{i}^{2} \leqslant 1+o(1)
$$

since $\varepsilon$ is arbitrary. Hypothesis $(\mathrm{H})$ is therefore established and the theorem follows from [8, Theorem 3.3 and Remark 3].

5. Finiteness of eigenvalues below $l_{e}$. For a form $t$ defined by (3.5) and (3.6), it will be necessary to define an associated form $t_{k}$ on $\Omega \cap \Omega_{k}$ for some $k \in \mathbf{N}$. The form $t_{k}$ is given by

$$
\begin{aligned}
t_{k}[u, v]= & \sum_{j=0}^{m} \int_{\Omega \cap \Omega_{k}}\left\langle A_{j} \nabla^{j} u, \nabla^{j} v\right\rangle d x \\
& +\sum_{j=0}^{m-1}\left\{\int_{\Gamma_{R} \cap \bar{\Omega}_{k}} \sigma_{j}\left(\frac{\partial}{\partial \nu}\right)^{j} u\left(\frac{\partial}{\partial \nu}\right)^{j} \bar{v} d s-\int_{\partial \Omega_{k} \cap \bar{\Omega}} \rho_{j}\left\langle\nabla^{j} u, \nabla^{j} v\right\rangle d s\right\}
\end{aligned}
$$

with domain $\mathscr{D}\left(t_{k}\right)=\left\{u: u=\phi \uparrow_{\Omega \cap \Omega_{k}}, \phi \in \mathscr{D}(t)\right\}$. 
Lemma 4. Assume (A)-(D). For some $k \in \mathbf{N}$ let $t_{k}$ be defined by (5.1) where each $\rho_{j} \in L^{\beta_{0}}\left(\partial \Omega_{k} \cap \Omega\right)$ and $\beta_{0}$ is given by (3.8) for $m=1$. Then, $t_{k}$ is bounded below and closable in $L_{w}^{2}\left(\Omega \cap \Omega_{k}\right)$.

Proof. By extending each $\rho_{j}$ as zero on $\partial \Omega \cap \Omega_{k}$ we may regard $\rho_{j}$ as being in $L^{\beta_{0}}\left(\partial\left(\Omega_{k} \cap \Omega\right)\right)$. As in the proof of Lemma 1 , given any $\varepsilon>0$ we may write $\rho_{j}=\rho_{j, 1}+\rho_{j, 2}$ where $\left|\rho_{j, 2}\right|<K(\varepsilon)$ on $\partial\left(\Omega_{k} \cap \Omega\right)$ and $\left\|\rho_{j, 1}\right\|_{L^{\beta_{0}\left(\partial\left(\Omega_{k} \cap \Omega\right)\right)}}<\varepsilon$. Then by Hölder's inequality with $p_{0}$ given by (2.2) (with $m=1$ ), applying (2.3) and (2.4) to $\gamma_{0}\left(\nabla^{j} \phi\right)$, and using (2.9)

$$
\begin{gathered}
\int_{\partial\left(\Omega_{k} \cap \Omega\right)}\left|\rho_{j}\right|\left|\nabla^{j} \phi\right|^{2} d s \leqslant \varepsilon\left\|\nabla^{j} \phi\right\|_{L^{p_{0}\left(\partial\left(\Omega_{k} \cap \Omega\right)\right)}}^{2}+K(\varepsilon)\left\|\nabla^{j} \phi\right\|_{\partial\left(\Omega_{k} \cap \Omega\right)}^{2} \\
\leqslant \varepsilon_{1}\left\|\nabla^{j} \phi\right\|_{1, \Omega_{k} \cap \Omega}^{2}+K(\varepsilon)\left(\varepsilon_{2}\left\|\nabla^{2} \phi\right\|_{1, \Omega_{k} \cap \Omega}^{2}+K\left(\varepsilon_{2}\right)\left\|\nabla^{j} \phi\right\|_{\left.\Omega_{k} \cap \Omega\right)}^{2}\right. \\
\leqslant \varepsilon_{3}\left\|\nabla^{m} \phi\right\|_{\Omega_{k} \cap \Omega}^{2}+K\left(\varepsilon_{3}\right)\|\phi\|_{\Omega_{k} \cap \Omega}^{2}
\end{gathered}
$$

for any $\phi \in \mathscr{D}\left(t_{k}\right)$. Finally, by (B)(1) and (D) we have that for any $\phi \in \mathscr{D}\left(t_{k}\right)$

$$
\int_{\partial \Omega_{k} \cap \Omega}\left|\rho_{j}\right|\left|\nabla^{j} \phi\right|^{2} d s \leqslant \varepsilon \int_{\Omega \cap \Omega_{k}} \mu_{m}\left|\nabla^{m} \phi\right|^{2} d x+K(\varepsilon) \int_{\Omega_{\cap} \cap \Omega_{k}} w|\phi|^{2} d x .
$$

Using (3.9), (3.10), and (5.2) we have that for any $\varepsilon \in(0,1)$, there is a constant $K_{0}(\varepsilon)$ such that

$$
t_{k}[u] \geqslant(1-\varepsilon) \int_{\Omega \cap \Omega_{k}} \mu_{m}\left|\nabla^{m} u\right|^{2} d x-K_{0}(\epsilon)\|u\|_{L_{w}^{2}\left(\Omega \cap \Omega_{k}\right)}^{2}
$$

for all $u \in \mathscr{D}\left(t_{k}\right)$. Therefore, $t_{k}$ is bounded below in $L_{w}^{2}\left(\Omega \cap \Omega_{k}\right)$.

The fact that $t_{k}$ is closable now follows from (5.3) and the proof of Lemma 2. This completes the proof of Lemma 4.

LEMMA 5. Let the form $t$ defined in (3.5) and (3.6) be bounded below and closable. Assume that for some integer $r \in \mathbf{N}$ the hypothesis of Lemma 4 is satisfied. If

$$
t[\phi] \geqslant t_{r}[\phi], \quad \phi \in \mathscr{D}(t),
$$

then there is a finite dimensional subspace $M$ of $L_{w}^{2}(\Omega)$ such that $(\tilde{T} u, u) \geqslant 0$ for all $u \in \mathscr{D}(\tilde{T}) \cap M^{\perp}$.

Proof. By Lemma $4, t_{r}$ is closable. Let $\tilde{T}_{r}$ be the lower semibounded selfadjoint operator associated with the closure $\tilde{t}_{r}$ in $L_{w}^{2}\left(\Omega \cap \Omega_{r}\right)$. Let $H\left(\tilde{t}_{r}\right)$ denote the Hilbert space defined by $\mathscr{D}\left(\tilde{t}_{r}\right)$ and the inner product

$$
(u, v)_{\tilde{t}_{r}}=t_{r}[u, v]+\left(K_{0}+1\right)(u, v)_{\Omega \cap \Omega}
$$

for $K_{0}$ defined in (5.3). In view of (5.3) and (2.13), the embedding $H\left(\tilde{t}_{r}\right) \rightarrow$ $W^{m, 2}\left(\Omega \cap \Omega_{r}\right)$ is continuous. Hence, from (A)(2), $H\left(\tilde{t}_{r}\right)$ is compactly embedded in $L_{w}^{2}\left(\Omega \cap \Omega_{r}\right)$. Consequently, $\tilde{T}_{r}$ has a compact resolvent and thus a discrete spectrum consisting of isolated eigenvalues of finite multiplicity; we denote these by

$$
\nu_{1} \leqslant \nu_{2} \leqslant \cdots \leqslant \nu_{N} \leqslant 0 \leqslant \nu_{N+1} \leqslant \cdots \rightarrow \infty,
$$


each eigenvalue being repeated according to multiplicity. Let $\left\{v_{l}: l \in \mathbf{N}\right\}$ be an associated orthonormal sequence of eigenfunctions. By setting them to be zero outside $\Omega \cap \Omega_{r}$ the functions $v_{l}$ can be considered to be in $L_{w}^{2}(\Omega)$.

Let $M$ be the linear span of $\left\{v_{1}, \ldots, v_{N}\right\}$ in $L_{w}^{2}(\Omega)$ and let $u \in \mathscr{D}(\tilde{T}) \cap M^{\perp}$. We claim that since $u \in \mathscr{D}(\tilde{t})$, then (5.4) is satisfied with $\phi=u$. To verify this we need to show that if $\left\{\phi_{l}\right\}$ is $\tilde{t}$-convergent to $u$ then $\left\{\phi_{l} \uparrow \Omega_{r} \cap \Omega\right\}$ is $\tilde{t}_{r}$-convergent to $u\left\lceil\Omega_{r} \cap \Omega\right.$. Clearly, $\phi_{l} \rightarrow u$ in $L_{w}^{2}(\Omega)$ implies that $\phi_{l} \uparrow \Omega_{r} \cap \Omega \rightarrow u \uparrow \Omega_{r} \cap \Omega$ in $L_{w}^{2}\left(\Omega \cap \Omega_{r}\right)$. Now, by (5.3) $t_{r}$ is bounded below in $L_{w}^{2}\left(\Omega \cap \Omega_{r}\right)$. Hence, by (5.4), $t\left[\phi_{l}-\phi_{m}\right] \rightarrow 0$ as $l, m \rightarrow \infty$ implies that $t_{r}\left[\phi_{l}-\phi_{m}\right] \rightarrow 0$. Therefore, $u \uparrow_{\Omega_{r} \cap \Omega} \in \mathscr{D}\left(\tilde{t}_{r}\right)$ and (5.4) holds for any $u \in \mathscr{D}(\tilde{T}) \cap M^{\perp}$.

By the min-max principle

$$
\nu_{N+1}=\sup _{\left\{\phi_{1}, \ldots, \phi_{N}\right\}} \inf \tilde{t}_{r}[\psi, \psi]
$$

where the infimum is over all $\psi \in \mathscr{D}\left(\tilde{t}_{r}\right) \cap\left[\phi_{1}, \ldots, \phi_{N}\right]^{\perp}$ with $\|\psi\|_{\Omega \cap \Omega_{r}}=1$. Whence, for any $u \in \mathscr{D}(\tilde{T}) \cap M^{\perp}$

$$
(\tilde{T} u, u)=\tilde{t}[u] \geqslant \tilde{t}_{r}[u] \geqslant \nu_{N+1}\|u\|_{L_{w}^{2}\left(\Omega \cap \Omega_{r}\right)}^{2} \geqslant 0,
$$

which completes the proof.

Theorem 6. Assume (A)-(D), and that $t$ defined in (3.5) and (3.6) is bounded below and closable. If for $r \in \mathbf{N}$ sufficiently large, there are functions

$$
\rho_{j} \in L^{\beta_{0}}\left(\partial \Omega_{r} \cap \Omega\right), \quad j=0,1, \ldots, m-1,
$$

such that for some number $\lambda$ and all $\phi \in \mathscr{D}(t)$

$$
\begin{aligned}
\sum_{j=0}^{m} \int_{\Omega \backslash \Omega_{r}} & \left\langle A_{j} \nabla^{j} \phi, \nabla^{j} \phi\right\rangle d x \\
& +\sum_{j=0}^{m-1}\left\{\int_{\Gamma_{R} \backslash \Omega_{r}} \sigma_{j}\left|\left(\frac{\partial}{\partial \nu}\right)^{j} \phi\right|^{2} d s+\int_{\partial \Omega_{r} \cap \Omega} \rho_{j}\left|\nabla^{j} \phi\right|^{2} d s\right\} \\
\geqslant & \lambda \int_{\Omega \backslash \dot{\Omega}_{r}} w|\phi|^{2} d x,
\end{aligned}
$$

then the number $N_{\lambda}(\tilde{T})$ of eigenvalues of $\tilde{T}$ below $\lambda$ is finite. Moreover, $N_{\lambda}(\tilde{T}) \leqslant$ $N_{\lambda}\left(\tilde{T}_{r}\right)$ where $\tilde{T}_{r}$ is the operator determined by $\tilde{t}_{r}$.

PROOF. The proof follows directly from Lemma 5 and the Spectral Theorem (cf. Glazman [12, p. 15]).

In the case of $\tau=-\Delta+q(x), x \in \mathbf{R}^{n}$, Piepenbrink [19] showed that the conclusion of Lemma 5 holds provided that

$$
-\Delta u+q u \geqslant 0, \quad x \in \mathbf{R}^{n},
$$

has a positive $C^{1}$-solution $v$ in $\{x:|x|>R\}$ for some $R>0$. The approach above extends the proof of Piepenbrink. Inequality (5.5) is closely related to inequality (4.2) in [19]. The function $\rho_{0}$ is given by $v^{-1}(\partial v / \partial \nu)$ in Piepenbrink's inequality (4.2) where $v$ is the positive solution required in his hypothesis. Allegretto [3] in the same year published a paper which showed the equivalence of two types of oscillatory 
behavior of solutions of $-\Delta u+q u=0, x \in \mathbf{R}^{n}$. Consequently, a critical question regarding the existence of positive solutions to the above equation was settled. These two papers showed an important connection between the positivity of solutions of these equations and the finiteness of the spectrum below $l_{e}$. This connection was developed in subsequent papers - (see Simon [31]) - by Allegretto [5-7], Piepenbrink [20, 21], and Moss and Piepenbrink [16]. However, this approach via oscillation theory seems to be limited particularly to second-order operators with Dirichlet boundary conditions. In this paper, the functions $\rho_{j}, j=0,1, \ldots, m-1$, arise from an inequality [15], which is related to the Uncertainty Principle Lemma (cf. [23, p. $169])$, rather than a positive solution.

6. Applications. In this section we illustrate some applications of Theorems 3 and 6 with particular emphasis given to the Schrodinger operator and, more generally, second-order operators. Rather general applications are first given for the sake of completeness, then we present some more specific examples.

We find lower bounds for $l_{e}\left(\tilde{T}_{2}\right)$, the least point of the essential spectrum, in terms of the coefficients of the operator $\tilde{T}_{2}$ defined below and the weight $w(x)$. Criteria are given concerning the coefficients which imply that the number of eigenvalues below $l_{e}\left(\tilde{T}_{2}\right)$ is finite. Finally, applications are given which apply to higher-order elliptic operators.

For clarity we respecify our operator and assumptions in this case. In the case of $m=1$, we write expression (3.1) as

$$
\tau_{2}:=w(x)^{-1}\left[-\sum_{i, j=1}^{n} \frac{\partial}{\partial x_{j}}\left(a_{i j}(x) \frac{\partial}{\partial x_{i}}\right)+q(x)\right], \quad x \in \Omega .
$$

The $n \times n$ matrix $A(x):=\left(a_{i j}(x)\right)$ for $i, j=1, \ldots, n$. The form associated with (6.1) is

$$
t_{2}[u, v]=\int_{\Omega}[\langle A \nabla u, \nabla u\rangle+q u \bar{v}] d x+\int_{\Gamma_{R}} \sigma(s) u(s) \bar{v}(s) d s
$$

for

$$
u, v \in \mathscr{D}\left(t_{2}\right)=\left\{u: u=\phi \uparrow_{\Omega}, \phi \in C_{0}^{\infty}\left(\mathbf{R}^{n} \backslash \Gamma_{S}\right)\right\} .
$$

The operator which is (formally) associated with $t$ is defined by $T_{2} u=\tau_{2} u, T_{2}$ : $\mathscr{D}\left(T_{2}\right) \rightarrow L_{w}^{2}(\Omega)$, with the dense domain

$$
\begin{aligned}
& \mathscr{D}\left(T_{2}\right)=\left\{u: u=\phi \prod_{\Omega}, \phi \in C_{0}^{\infty}\left(\mathbf{R}^{n} \backslash \Gamma_{S}\right), \tau_{2} u \in L_{w}^{2}(\Omega)\right. \text { and } \\
& \left.c(s) \partial \phi(s) / \partial \nu_{A}+\sigma(s) \phi(s)=0, s \in \Gamma_{R}\right\}
\end{aligned}
$$

where $\partial \phi / \partial \nu_{A}=(\nabla \phi)^{\dagger} A \cdot \nu$ with $\nu$ being the unit outward normal derivative on $\Gamma_{R}$. Here $c(s)$ and $\sigma(s)$ are assumed not to be simultaneously zero on $\Gamma_{R}$. Hence, $T_{2}$ is associated with a mixed boundary value problem on $\Omega$.

The assumptions (A)-(D) of $\S 3$ are simplified (and slightly modified) as follows for the second-order case:

(a) For the $\Omega_{k}$ 's defined in (2.12) we require that $\partial\left(\Omega \cap \Omega_{k}\right)$ be of class $C^{1}$ for each positive integer $k$. 
(b) The matrix $A(x)$ is Hermitian a.e. on $\Omega$,

$$
\mu_{A}(x):=\min \text { e.v. } A(x)>0 \text { for a.e. } x \in \Omega,
$$

and $\mu_{A}(x)^{-1} \in L^{\infty}\left(\Omega \cap \Omega_{k}\right)$ for each $k \in \mathbf{N}$.

The potential $q(x)$ is real-valued in $\Omega$,

$$
\lim _{k \rightarrow \infty} \inf _{x \in \Omega \backslash \Omega_{k}}\left\{\frac{q(x)}{w(x)}\right\}=: q_{0}>-\infty,
$$

and for each $k \in \mathbf{N}, q \in L^{\alpha_{0}}\left(\Omega \cap \Omega_{k}\right)$ where $\alpha_{0}=n / 2$ for $n>2$ and $\alpha_{0}>1$ for $n=2$.

(c) (1) The real-valued function $\sigma \in L^{\beta_{0}}\left(\Gamma_{R}\right)$ where $\beta_{0}=n-1$ for $n>2$ and $\beta_{0}>1$ for $n=2$.

(2) There is a $k_{0} \in \mathbf{N}$ such that $\sigma \geqslant 0$ in $\Gamma_{R} \backslash \bar{\Omega}_{k}$ for all $k \geqslant k_{0}$.

(d) The weight $w>0$ a.e. on $\Omega$, and $w^{-1} \in L^{\infty}\left(\Omega \cap \Omega_{k}\right), k \in \mathbf{N}$.

For the sake of simplification we further assume

(e) There are Dirichlet boundary conditions at $\infty$, i.e., $\phi(s)=0, s \in \Gamma_{R} \backslash \Omega_{k}$, for some $k$ sufficiently large and all $\phi \in \mathscr{D}(t)$.

This condition can be removed in some cases, as is shown in [14]. An example of how this is done is given in Corollaries 14 and 15.

By Lemma 2, the assumptions (a)-(d) imply that the form $t$ is bounded below and closable. Analogously to $\tilde{T}$ in $\S 4$ we let $\tilde{T}_{2}$ be the unique, lower semibounded, selfadjoint operator associated with the closure of $t_{2}$. When the form $t_{2}$ arises from $T_{2}$ as in (3.4), $\tilde{T}_{2}$ will be the Friedrichs extension of $T_{2}$.

A crucial ingredient in the applications to follow will be a Friedrichs-type inequality which is the next lemma. The lemma is a generalization of the Uncertainty Principle Lemma [23, p. 169]. A proof can be found in [14 and 15]. The set $G \subseteq \mathbf{R}^{n}$ is open and connected with a boundary $\partial G$ which is smooth enough in order that the first formula of Green applies.

LEMMA 7. Let $g$ be an element of $W^{2,2}(G)$ whose Laplacian $\Delta g$ is of one sign a.e. on G. Define $\eta=1$ if $\Delta g<0$ a.e. on $G$ and $\eta=0$ if $\Delta g>0$ a.e. on $G$. If $\left(\Sigma_{|\alpha|=j}\left|D^{\alpha} \phi\right|^{2}\right)^{1 / 2} \in W^{1,2}(G)$ then

$$
\begin{aligned}
\int_{G}|\Delta g| \sum_{|\alpha|=j}\left|D^{\alpha} \phi\right|^{2} d x \leqslant & (-1)^{\eta} \int_{\partial G} \gamma_{1}(g) \gamma_{0}\left(\sum_{|\alpha|=j}\left|D^{\alpha} \phi\right|^{2}\right) d s \\
& +2 \int_{G}|\nabla g| \sum_{|\alpha|=j}\left|D^{\alpha} \phi\right|\left|\nabla D^{\alpha} \phi\right| d x \\
\leqslant & (-1)^{\eta} 2 \int_{\partial G} \gamma_{1}(g) \gamma_{0}\left(\sum_{|\alpha|=j}\left|D^{\alpha} \phi\right|^{2}\right) d s \\
& +4 \min \{n, j+1\} \int_{G}|\Delta g|^{-1}|\nabla g|^{2} \sum_{|\alpha|=j+1}\left|D^{\alpha} \phi\right|^{2} d x .
\end{aligned}
$$

This Friedrichs-type inequality plays here in the higher dimensional case the role of the related Hardy inequality in the one dimensional case (e.g. see [12]). 
THEOREM 8. Let (a)-(e) hold and suppose that each $a_{i j} \in L^{\infty}\left(\Omega \cap \Omega_{k}\right), k \in \mathbf{N}$. Assume that for some $k \in \mathbf{N}$ there are functions $g, h \in W^{2,2}\left(\Omega \backslash \Omega_{k}\right)$ which satisfy the following:

(i) $\Delta g$ and $\Delta h$ are each of one sign a.e. on $\Omega \backslash \Omega_{k}$,

(ii) $\mu_{A}(x) \geqslant|\nabla g|^{2} /|\Delta g|$ for a.e. $x \in \Omega \backslash \Omega_{k}$, and

(iii) $w(x) \leqslant|\Delta h|$ a.e. $x \in \Omega \backslash \Omega_{k}$.

Then

$$
l_{e}\left(\tilde{T}_{2}\right) \geqslant \lim _{k \rightarrow \infty}\left[4^{-1} \inf _{x \in \Omega \backslash \Omega_{k}} \frac{|\nabla g|}{|\nabla h|}+\inf _{x \in \Omega \backslash \Omega_{k}} \frac{q}{w}\right] .
$$

Proof. Let

$$
\sum=\lim _{k \rightarrow \infty} \inf _{x \in \Omega \backslash \Omega_{k}}|\nabla g| /|\nabla h| \text { and } q_{0}=\lim _{k \rightarrow \infty} \inf _{x \in \Omega \backslash \Omega_{k}} q / w .
$$

By Theorem 3 it will suffice to show that for each arbitrarily small $\delta>0$, there is a $K \in \mathbf{N}$ such that for any $k \geqslant K$

$$
t[u] \geqslant\left(\sum+q_{0}-\delta\right)\|u\|_{L_{w}^{2}(\Omega)}^{2} \text { for all } u \in \mathscr{D}(t)
$$

with supp $u \subset \Omega \backslash \Omega_{k}$. We may assume that $q_{0}-\delta$ is nonnegative, since the addition of a constant multiplicative operator to $\tilde{T}_{2}$ will only shift $l_{e}\left(T_{2}\right)$ by that same constant amount. For a given $\delta>0, k$ sufficiently large, and supp $u \subset \Omega \backslash \Omega_{k}$,

$$
\begin{aligned}
t[u] & \geqslant \int_{\Omega \backslash \Omega_{k}}\left[\frac{|\nabla g|^{2}|\nabla u|^{2}}{|\Delta g|}+\left(q_{0}-\frac{\delta}{2}\right) w|u|^{2}\right] d x \\
& \geqslant \int_{\Omega \backslash \Omega_{k}}\left[2^{-1}|\nabla g||\nabla u||u|+\left(q_{0}-\frac{\delta}{2}\right) w|u|^{2}\right] d x \\
& \geqslant 2^{-1}\left(\sum-\frac{\delta}{2}\right) \int_{\Omega \backslash \Omega_{k}}|\nabla h||\nabla u||u| d x+\left(q_{0}-\frac{\delta}{2}\right)\|u\|_{L_{w}^{2}(\Omega)}^{2} \\
& \geqslant\left(4^{-1} \sum+q_{0}-\delta\right)\|u\|_{L_{w}^{2}(\Omega)}^{2}
\end{aligned}
$$

by Lemma 7, which completes the proof.

As illustrated by the examples below, Theorem 8 is sharp in certain cases. We emphasize the fact that the conditions imposed on the coefficients are only needed in some neighborhood of the singularities.

Before considering more specific examples of applications of Theorem 6, we continue with a related corollary of Theorem 6. (Recall that $f^{-}(x)=f(x)$ when $f(x) \leqslant 0$ and $f^{-}(x)=0$ when $f(x)>0$.)

THEOREM 9. Let (a)-(e) be satisfied. Assume that for some $r \in \mathbf{N}$ there are functions $f, g \in W^{2,2}\left(\Omega \backslash \Omega_{r}\right)$ which satisfy the following for some real number $\lambda$ :

(i) $\mu_{A}(x) \geqslant|\nabla g|^{2} /|\Delta g|$ for a.e $x \in \Omega \backslash \Omega_{r}$,

(ii) $[q(x)-\lambda w(x)]^{-} \geqslant \Delta$ ffor a.e. $x \in \Omega \backslash \Omega_{r}$,

(iii) $\Delta g$ is of one sign on $\Omega \backslash \Omega_{r}$,

(iv) $\rho \in L^{\beta_{0}}\left(\partial \Omega_{r} \cap \Omega\right)$ for $\rho:=(-1)^{\eta} 4^{-1} \gamma_{1}(g)-\gamma_{1}(f)$ where $\eta=1$ if $\Delta g<0$ and $\eta=0$ if $\Delta g>0$ in $\Omega \backslash \Omega_{r}$. 
If

$$
\lim _{k \rightarrow \infty} \sup _{x \in \Omega \backslash \Omega_{k}} \frac{|\nabla f|}{|\nabla g|}<\frac{1}{4},
$$

then $\tilde{T}_{2}$ has only a finite number of eigenvalues below $\lambda$.

Proof. For $\delta \in\left(0, \frac{1}{4}\right)$ choose $k \in \mathbf{N}$ large enough such that $k \geqslant r$ and $|\nabla f| \leqslant$ $\left(4^{-1}-\delta\right)|\nabla g|$ for a.e. $x \in \Omega \backslash \Omega_{k}$. If $\phi \in \mathscr{D}\left(t_{2}\right)$, then by Lemma 7

$$
\begin{array}{rl}
-\int_{\Omega \backslash \Omega_{k}}[q-\lambda w]^{-}|\phi|^{2} & d x \leqslant \int_{\Omega \backslash \Omega_{k}}|\Delta f||\phi|^{2} d x \\
\leqslant & -\int_{\partial\left(\Omega \backslash \Omega_{k}\right)} \gamma_{1}(f)|\phi|^{2} d s+2 \int_{\Omega \backslash \Omega_{k}}|\nabla f||\nabla \phi||\phi| d x \\
\leqslant & -\int_{\partial\left(\Omega \backslash \Omega_{k}\right)} \gamma_{1}(f)|\phi|^{2} d s+\left(2^{-1}-2 \delta\right) \int_{\Omega \backslash \Omega_{k}}|\nabla g||\nabla \phi||\phi| d x \\
\leqslant & \int_{\partial \Omega_{k} \cap \Omega}\left(\rho-(-1)^{\eta} \delta \gamma_{1}(g)\right)|\phi|^{2} d s \\
& +(1-4 \delta) \int_{\Omega \backslash \Omega_{k}}|\Delta g|^{-1}|\nabla g|^{2}|\nabla \phi|^{2} d x .
\end{array}
$$

Since $\delta$ can be chosen to be arbitrarily small, the above series of inequalities and the hypothesis imply that (5.5) holds. Thus, the proof is completed.

Note that conditions (e) can be removed as a requirement in Theorem 9 if we know that $\sigma-\rho \geqslant 0$ on $\Gamma_{R} \backslash \bar{\Omega}_{r}$ for some $r$. (See Corollary 14.) The problem now becomes that of finding functions $f, g$, and $h$ which satisfy the conditions of Theorems 8 and 9. A natural hope is that of approximating the coefficients and weight by radial functions, i.e., functions of $|x|$, in a neighborhood of the singularities. In some situations it might be more realistic to approximate the coefficients and weight in a neighborhood of the finite singularities, $S \backslash\{\infty\}$, by a function of the distance from $x$ to $S \backslash\{\infty\}$. More generally, we might assume our approximating functions-hence, $f, g$, and $h$-to be functions of a single variable $r$ which is a function of $x$. For example, if we assume that $h=h(r(x))$, then the inequality (iii) of Theorem 8 is satisfied if

$$
w(x) \leqslant \omega(r) \leqslant|\Delta h|
$$

which implies that

$$
\omega(r) \leqslant\left.\left|h^{\prime \prime}(r)\right| \nabla r\right|^{2}+h^{\prime}(r) \Delta r \mid .
$$

If $g=g(r(x))$ then inequality (ii) is satisfied if $\mu_{A}(x) \geqslant \tilde{\mu}(r) \geqslant|\nabla g|^{2} /|\Delta g|$ and

$$
\left.\left|g^{\prime \prime}(r)\right| \nabla r\right|^{2}+\left.g^{\prime}(r) \Delta r\left|\tilde{\mu}(r) \geqslant\left[g^{\prime}(r)\right]^{2}\right| \nabla r\right|^{2} .
$$

In this case the ratio $|\nabla g| /|\nabla h|$ of Theorem 8 reduces to $\left|g^{\prime}(r)\right| /\left|h^{\prime}(r)\right|$, provided $|\nabla r| \neq 0$. For Theorem 9 the situation is analogous.

When the portion of the singular set which is of interest is a point-say $s \in S$-then we may let $r(x)=|x-s|$. If the singularity is at $\infty$, then $r(x)=|x|$ is usually appropriate. In either case $|\nabla r| \equiv 1$ and $\Delta r=(n-1) / r$ for which (6.5) and 
(6.6) can be solved easily. In the case that the singular set includes a hyperplane-say $s_{j} \equiv 0$ - then $r(x)=\left|x_{j}-s_{j}\right|$ is appropriate. Here, $\Delta r \equiv 0$ and $|\nabla r| \equiv 1$. Again, (6.5) and (6.6) follow readily. Finally, suppose that the singular set includes a portion of the surface of a ball $B\left(x_{0} ; \rho\right)$ with center $x_{0} \in \mathbf{R}^{n}$ and radius $\rho>0$. Further assume that the exterior of the ball intersects $\Omega$ with $\partial B \cap \partial \Omega \neq \varnothing$. In this case the distance $r(x)$ from a point $x \in \Omega$ (near $\partial B$ ) to $\partial B$ is given by $r(x)=\mid x-$ $x_{0} \mid-\rho$. Here, $|\nabla r|=1$ and $\Delta r=(n-1) /\left|x-x_{0}\right|=(n-1)(r+\rho)$ from which (6.5) and (6.6) can be solved. (The singular set $S$ may even be formed by an unbounded sequence of such balls.)

More generally, (6.5) and (6.6) are easier to solve if we know a priori that $|\nabla r| \geqslant 1$ and either

$$
\Delta r \geqslant \beta(r)|\nabla r|^{2}, \quad x \in \Omega \backslash \Omega_{k}
$$

or

$$
\Delta r \leqslant \beta(r)|\nabla r|^{2}, \quad x \in \Omega \backslash \Omega_{k},
$$

for some function $\beta(r)$. In the case of (6.7) and (6.8), it is possible to reduced (6.5) and (6.6) to linear differential inequalities in $h^{\prime}$ and $g^{\prime}$.

In the case that $r$ represents the distance from $x$ to $S \backslash\{\infty\}$ in some neighborhood of the singularities, it appears that (6.7) might be a condition concerning the "shape" of $\partial \Omega \cap S$ in that neighborhood. But, exactly what that condition might be remains open. Of course, inequalities of this type have been studied by many investigators, (e.g. see the paper of Redheffer [22]).

We summarize these observations with the following corollaries to Theorems 8 and 9 as well as some examples.

COROLlARY 10. Let (a)-(e) hold and suppose that each $a_{i j} \in L^{\infty}\left(\Omega \cap \Omega_{k}\right), k \in \mathbf{N}$. Assume that for $k$ sufficiently large:

(i) there is a function $r(x) \in W^{2,2}\left(\Omega \backslash \Omega_{k}\right)$ such that (6.7) holds for some function $\beta$ and constant $c>0$ for which $\int_{c}^{t} \beta(v) d v$ is absolutely continuous on $(0, \infty)$;

(ii) there is a function $\tilde{\mu}$ such that $\int_{t}^{d} \tilde{\mu}(s)^{-1} \exp \left(-\int_{c}^{s} \beta(v) d v\right) d s$ is absolutely continuous on $(0, \infty)$ and $\mu_{A}(x) \geqslant \tilde{\mu}(r(x))$ for $x \in \Omega \backslash \Omega_{k}$;

(iii) there is a function $\tilde{w}$ such that for some number $d>0$

$$
\int_{d}^{t} \tilde{w}(s) \exp \left(\int_{d}^{s} \beta(v) d v\right) d s
$$

is absolutely continuous on $(0, \infty)$ and $w(x) \leqslant \tilde{w}(r(x))$ for $x \in \Omega \backslash \Omega_{k}$.

Then

$$
\begin{aligned}
l_{e}\left(\tilde{T}_{2}\right) \geqslant \lim _{k \rightarrow \infty}\left\{4^{-1} \inf _{x \in \Omega \backslash \Omega_{k}}\right. & {\left[\int_{d}^{r(x)} \tilde{w}(t) \exp \left(\int_{d}^{t} \beta(s) d s\right) d t\right.} \\
& \left.\times \int_{r(x)}^{c} \tilde{\mu}(t)^{-1} \exp \left(-\int_{c}^{t} \beta(s) d s\right) d t\right]^{-1} \\
& \left.+\inf _{x \in \Omega \backslash \Omega_{k}} \frac{q}{w}\right\} .
\end{aligned}
$$


Proof. Using (6.5) and (6.6) we define

$$
h(r)=\int_{d}^{r} \exp \left(-\int_{d}^{t} \beta(v) d v\right) \int_{d}^{t} \tilde{w}(s) \exp \left(\int_{d}^{s} \beta(v) d v\right) d s d t
$$

and

$$
g(r)=\int_{c}^{r} \exp \left(-\int_{c}^{t} \beta(v) d v\right)\left[\int_{t}^{c} \tilde{\mu}(s)^{-1} \exp \left(-\int_{c}^{s} \beta(v) d v\right) d s\right]^{-1} d t .
$$

Now, $g(r(x))$ and $h(r(x))$ satisfy the hypothesis of Theorem 8 . Hence, the conclusion follows from (6.3).

COROllary 11. Let (a)-(e) hold. For $k$ sufficiently large assume (i) and (ii) of Corollary 10 and that for some real number $\lambda$ there is a function $\tilde{q}$ such that $\int_{d}^{t} \tilde{q}(s) \exp \left(\int_{d}^{s} \beta(v) d v\right) d s$ is absolutely continuous on $(0, \infty)$ and $[q(x)-\lambda w(x)]^{-} \geqslant$ $\tilde{q}(r(x))$ for a.e. $x \in \Omega \backslash \Omega_{k}$. If

$$
\begin{aligned}
\lim _{k \rightarrow \infty} \sup _{x \in \Omega \backslash \Omega_{k}} \int_{d}^{r(x)}|\tilde{q}(t)| \exp \left(\int_{d}^{t} \beta(s) d s\right) d t \\
\quad \times \int_{r(x)}^{c} \tilde{\mu}(t)^{-1} \exp \left(-\int_{c}^{t} \beta(s) d s\right) d t<\frac{1}{4},
\end{aligned}
$$

then $\tilde{T}_{2}$ has only a finite number of eigenvalues below $\lambda$.

Proof. Define $g$ as in the proof of Corollary 10. The definition of $f$ is the same as $h$ was defined in the proof of Corollary 10 except $|\tilde{q}|$ replaces $\tilde{w}$ in that definition. The proof now follows from Theorem 9.

When $r(x)=|x-s|$ for some $s \in S$, then (6.9) and (6.10) reduce to

$$
\begin{aligned}
l_{e}\left(\tilde{T}_{2}\right) \geqslant \lim _{k \rightarrow \infty}\left\{4^{-1} \inf _{x \in \Omega \backslash \Omega_{k}}\right. & {\left[\int_{d}^{|x-s|}|t-s|^{n-1} \tilde{w}(t) d t\right.} \\
& \left.\left.\times \int_{|x-s|}^{c}|t-s|^{1-n} \tilde{\mu}(t)^{-1} d t\right]^{-1}+\inf _{x \in \Omega \backslash \Omega_{k}} \frac{q}{w}\right\}
\end{aligned}
$$

and

$$
\begin{aligned}
\lim _{k \rightarrow \infty} \sup _{x \in \Omega \backslash \Omega_{k}} & \int_{d}^{|x-s|}|t-s|^{n-1}|\tilde{q}(t)| d t \\
& \times \int_{|x-s|}^{c}|t-s|^{1-n} \tilde{\mu}(t)^{-1} d t<\frac{1}{4}
\end{aligned}
$$

respectively.

EXAMPLE 12. Let $\Omega$ be an open, connected, unbounded subset of $\mathbf{R}^{n}$. Assume that $\Omega$ lies on one side of its boundary $\partial \Omega$, which is $C^{1}$ as a submanifold. Define the form

$$
h[u, v]=\int_{\Omega}[\langle A \nabla u, \nabla u\rangle+q u \bar{v}] d x+\int_{\partial \Omega} \sigma(s) u(s) \bar{v}(s) d s
$$

for all $u$ and $v$ in

$$
\mathscr{D}(h)=\left\{u: u=\phi \uparrow_{\Omega}, \phi \in C_{0}^{\infty}\left(\mathbf{R}^{n}\right), \operatorname{supp} \phi \cap N_{k} \Subset \Omega\right\}
$$


for some $k$ where $N_{k}=\left\{x \in \mathbf{R}^{n}:|x|>k\right\}$. We make the following assumptions regarding the coefficients.

(1) The matrix $A$ is Hermitian a.e. on $\Omega, \mu_{A}(x)>0$ a.e. on $\Omega$, and $\mu_{A}^{-1} \in$ $L^{\infty}\left(\Omega \backslash N_{k}\right)$ for each $k \in \mathbf{N}$.

(2) Let $w>0$ a.e. on $\Omega$ be a weight function for which $w^{-1} \in L^{\infty}\left(\Omega \backslash N_{k}\right)$ for each $k \in \mathbf{N}$.

(3) The potential $q(x)$ is real-valued in $\Omega$; for each $k \in \mathbf{N}, q \in L^{\alpha}\left(\Omega \backslash N_{k}\right)$ for $\alpha=n / 2$ when $n>2$ and $\alpha>1$ when $n=2$; and

$$
\lim _{|x| \rightarrow \infty} \inf _{|z| \geqslant|x|}\left\{\frac{q(z)}{w(z)}\right\}>-\infty .
$$

(4) The real-valued function $\sigma \in L^{\alpha}(\partial \Omega)$ and $\sigma \geqslant 0$ in $\partial \Omega \cap N_{k}$ for $k$ sufficiently large.

Let $T_{h}$ be the unique, lower semibounded, selfadjoint operator associated with the closure of $h$ in $L_{w}^{2}(\Omega)$. Assume that in some neighborhood $N_{K}$ of $\infty$ there exist piecewise continuous functions $\tilde{q}(t), \tilde{\mu}(t)$, and $\tilde{w}(t)$ of a real variable $t$ such that

(5) for some real number $\lambda,[q(x)-\lambda w(x)]^{-} \geqslant \tilde{q}(|x|), x \in \Omega \cap N_{K}$,

(6) $\mu_{A}(x) \geqslant \tilde{\mu}(|x|), x \in \Omega \cap N_{K}$, and

(7) $w(x) \leqslant \tilde{w}(|x|), x \in \Omega \cap N_{K}$.

If each $a_{i j} \in L^{\infty}\left(\Omega \backslash N_{k}\right), k \in \mathbf{N}$, then

$$
l_{e}(T) \geqslant \lim _{|x| \rightarrow \infty}\left\{4^{-1} \inf _{y \geqslant|x|}\left[\int_{d}^{y}|t|^{n-1} \tilde{w}(t) d t \int_{y}^{c}|t|^{1-n} \tilde{\mu}(t)^{-1} d t\right]^{-1}+\inf _{|z| \geqslant|x|} \frac{q(z)}{w(z)}\right\} .
$$

If

$$
\lim _{|x| \rightarrow \infty} \sup _{y \geqslant|x|}\left\{\int_{d}^{y}|t|^{n-1}|\tilde{q}(t)| d t \int_{y}^{c}|t|^{1-n} \tilde{\mu}(t)^{-1} d t\right\}<\frac{1}{4}
$$

then $T_{h}$ has only a finite number of eigenvalues below $\lambda$.

Proof. According to the conditions imposed on $\bar{\Omega}$ we may define each $\Omega_{k}$ in order that $\Omega_{k} \supset \Omega \backslash N_{k}$ and conditions (A)(1)-(A)(3) are met. Conditions (b)-(d) are met by (1)-(4) of the hypothesis. Corollaries 10 and 11 imply the conclusions above.

If the limit in (6.13) is $\infty$, then $T_{h}$ has a purely discrete spectrum. This is the conclusion of Theorem 1 in [14]. In fact, we have shown that condition (11) of Theorem 1 in [14] is unnecessary as was conjectured in that paper.

Consider the special case of (6.1) and Example 12 in which

$$
\tau_{2} u=-\Delta u+q(x) u, \quad x \in \Omega,
$$

and

$$
\begin{aligned}
u \in \mathscr{D}\left(\tau_{2}\right)=\{u: u= & \phi \uparrow_{\Omega}, \phi \in C_{0}^{\infty}\left(\mathbf{R}^{n}\right), \tau_{2} u \in L^{2}(\Omega) \text { and } \\
& c(s)(\partial \phi(s) / \partial \nu)+\sigma(s) \phi(s)=0, s \in \partial \Omega\},
\end{aligned}
$$


where $c(s)$ assumes only the values 0 and 1 is not simultaneously zero with $\sigma(s)$; furthermore, let $c(s)=0$ and $\sigma(s)=1$ for $s \in \partial \Omega \backslash N_{k}$ for some $k$. Here, $q$ and $\sigma$ are sufficiently smooth in order that (3) and (4) are true and

$$
t_{2}[u, v]=\left(\tau_{2} u, v\right), \quad u, v \in \mathscr{D}\left(\tau_{2}\right) \equiv \mathscr{D}\left(t_{2}\right) .
$$

(In this case $T_{h}$ is the Friedrichs extension of $\tau_{2}$.) Using (6.14) we have that for $n \geqslant 3, \lambda=0$, and $\tilde{q}(|x|) \leqslant q^{-}(x)$

$$
\lim _{|x| \rightarrow \infty} \inf _{y \geqslant|x|} y^{2-n} \int_{1}^{y} t^{n-1} \tilde{q}(t) d t>-\frac{n-2}{4}
$$

implies that $T_{h}$ has only a finite number of negative eigenvalues. This criterion compares favorably with the well-known criterion for the two-body problem (Theorem XIII.6 of [24]). Theorem 8 of Schmincke [30] concerning a condition, which implies that $T_{h}$ (with Dirichlet boundary conditions) has an infinite number of negative eigenvalues, provides an interesting contrast to condition (6.15).

We now proceed to a higher-order application. To do this we will need to first prove a corollary to the inequality in Lemma 7 (cf. Corollary 2 of [15]). Recall that $\nu(s)$ denotes the unit outward normal at $s,\left|\nabla^{j} \phi(x)\right|^{2}:=\sum_{|\alpha|=j}\left|D^{\alpha} \phi(x)\right|^{2}$, and $(s, v)$ is the Euclidean inner product with $\nu(s)$ of the position vector from the origin to $s \in \partial \Omega$.

COROLlARY 13. Let $G \subseteq \mathbf{R}^{n}$ be an open, connected set with a boundary $\partial G$ which is smooth enough in order that the first formula of Green applies. Let $\kappa$ be a real number. If the set $G$ contains the origin, then we restrict $\kappa>(2-n) / 2$. Define $\eta=1$ if $\kappa \in(2-n, 0)$ and $\eta=0$, otherwise. Then

$$
\begin{aligned}
\int_{G}|x|^{\kappa}\left|\nabla^{j} \phi\right|^{2} d x \geqslant & \frac{|\kappa-2+n|^{2}}{4 \min \{n, j\}} \int_{G}|x|^{\kappa-2}\left|\nabla^{j-1} \phi\right|^{2} d x \\
& +\frac{(-1)^{\eta}|\kappa-2+n|}{2 \min \{n, j\}} \int_{\partial G}(s, \nu)|s|^{k-2}\left|\nabla^{j-1} \phi\right|^{2} d s
\end{aligned}
$$

for all $\phi \in\left\{\phi: \phi=u \uparrow_{G}, u \in C_{0}^{\infty}\left(\mathbf{R}^{n}\right)\right\}$ and integers $j \geqslant 1$.

Proof. If $\kappa \neq 0$, let $g(x)=|x|^{\kappa}$ in Lemma 7. If $\kappa=0$ and $n>2$, let $g(x)=$ $4 \log |x| /(n-2)$. If $n=1,(6.16)$ is a form of the Hardy inequality.

We note that in the case that $0 \in G,(6.16)$ holds for all $\kappa$ if $\phi=u \uparrow_{G}$, $u \in C_{0}^{\infty}\left(\mathbf{R}^{n} \backslash\{0\}\right)$. Define $\Gamma^{+}$and $\Gamma^{-}$to be subsets of $\partial \Omega$ where the product $(s, \nu)$ is positive or nonpositive, respectively.

Corollary 14. Assume the following:

(i) the hypothesis of Lemma 2;

(ii) for some $j \in\{1, \ldots, m\}$, and some real number $\kappa$

$$
\lim _{k \rightarrow \infty} \inf _{x \in \Omega \backslash \Omega_{k}}|x|^{-\kappa} \mu_{j}(x)>b_{j}>0 ;
$$

(iii) there is an integer $k_{2}$ such that one of the following conditions is met:

(a) each $\phi \in \mathscr{D}(t)$ satisfies Dirichlet boundary conditions on $\partial \Omega \backslash \Omega_{k_{2}}$, or

(b) $n>2 j, \kappa \in(2 j-n, 0)$, and $D^{\alpha} \phi=0$ on $\Gamma^{+} \backslash \Omega_{k_{2}}$ for any $\phi \in \mathscr{D}(t)$ and $|\alpha|=0,1, \ldots, j-1$, or 
(c) $\kappa \in(-\infty, 2-n] \cup[2 j-2, \infty)$, and $D^{\alpha} \phi=0$ on $\Gamma^{-} \backslash \Omega_{k_{2}}$ for any $\phi \in$ $\mathscr{D}(t)$ and $|\alpha|=0,1, \ldots, j-1$;

(iv) if $0 \in \bar{\Omega} \backslash \Gamma_{S}$, we further restrict $2 \kappa>4 j-n-2$.

If

$$
\lim _{k \rightarrow \infty} \inf _{x \in \Omega \backslash \Omega_{k}} \frac{B(j)|x|^{k-2 j}+A_{0}(x)}{w(x)}>\lambda
$$

where

$$
B(j)=b_{j} 4^{-j} \prod_{i=1}^{j} \frac{|\kappa-2 i+n|}{\min \{n, j+1-i\}},
$$

then $\sigma(\tilde{T}) \cap(-\infty, \lambda)$ is finite.

Proof. Choose $r \geqslant \max \left\{k_{0}, k_{1}, k_{2}\right\}$ (for $k_{1}$ defined in Lemma 2 and $k_{0}$ defined in (C)(2)) in order that $\mu_{j}(x) \geqslant b_{j}|x|^{\kappa}$ and

$$
B(j)|x|^{\kappa-2 j}+A_{0}(x) \geqslant \lambda w(x), \quad x \in \Omega \backslash \Omega_{r} .
$$

By (i), (C)(2), and Corollary 13 , for any $\phi \in \mathscr{D}(t)$

$$
\begin{aligned}
& \sum_{j=0}^{m} \int_{\Omega \backslash \Omega_{r}}\left\langle A_{j} \nabla^{j} \phi, \nabla^{j} \phi\right\rangle d x+\sum_{j=0}^{m-1} \int_{\partial \Omega \backslash \Omega_{r}} \sigma_{j}\left|\left(\frac{\partial}{\partial \nu}\right)^{j} \phi\right|^{2} d s \\
& \quad \geqslant \int_{\Omega \backslash \Omega_{r}}\left\{b_{j}|x|^{\kappa}\left|\nabla^{j} \phi\right|^{2}+A_{0}(x)|\phi|^{2}\right\} d x \\
& \quad \geqslant \int_{\Omega \backslash \Omega_{r}}\left[B(j)|x|^{\kappa-2 j}+A_{0}(x)\right]|\phi|^{2} d x+\sum_{l=1}^{j} I_{l} \\
& \quad \geqslant \lambda \int_{\Omega_{\backslash} \Omega_{r}} w|\phi|^{2} d x+\sum_{l=1}^{j} I_{l}
\end{aligned}
$$

where there are constants $C_{l}$ according to (6.16) such that

$$
I_{l}=C_{l} \int_{\partial\left(\Omega \backslash \Omega_{r}\right)}(s, \nu)|s|^{\kappa-2 l}\left|\nabla^{j-l} \phi\right|^{2} d s, \quad l=1, \ldots, j .
$$

Let $\rho_{j-l}(s)=-C_{l}(s, \nu)|s|^{\alpha-2 l}$ for $s \in \partial \Omega_{r} \cap \Omega$ and $l=1, \ldots, j$. Otherwise, set $\rho_{i}(s) \equiv 0$ for $j \leqslant i \leqslant m-1$. By choosing $r$ properly, we can be assured that $0 \notin \partial \Omega_{r} \cap \Omega$. Hence, each $\rho_{i} \in L^{\beta_{0}}\left(\partial \Omega_{r} \cap \Omega\right), 0 \leqslant i \leqslant m-1$. The sign of $I_{l}$ over $\partial \Omega \backslash \Omega$, depends on $\kappa$ and the angle between the position vector from the origin to $s$ and the unit outward normal $\nu$ at $s$. By assumptions (iii) and (iv) above this portion of $I_{l}$ is nonnegative. The hypothesis of Theorem 6 is now satisfied and the conclusion follows.

Note that in Corollary 14 we are not necessarily assuming Dirichlet conditions at infinity-see (iii).

For $\tau_{2}=-\Delta+q$, above we would let $m=1, j=1, w(x) \equiv 1$, and $\kappa=0$. Then, the corresponding fact for the 2-body problem follows as in Theorem XIII.6(b) of Reed and Simon [24]. 
COROllary 15. Assume the hypothesis of Theorem 3 , that for some $j \in\{1, \ldots, m\}$

$$
\lim _{k \rightarrow \infty} \inf _{x \in \Omega \backslash \Omega_{k}}|x|^{-\kappa} \mu_{j}(x) \geqslant b_{j}>0
$$

for some real number $\kappa$, the hypothesis (iii) and (iv) of Corollary 14. Then, for $B(j)$ defined in Corollary 14

$$
l_{e}(\tilde{T}) \geqslant \lim _{k \rightarrow \infty} \inf _{x \in \Omega \backslash \Omega_{k}} \frac{B(j)|x|^{\kappa-2 j}+A_{0}(x)}{w(x)} .
$$

Proof. Using Theorem 3, this proof follows similarly to the proof of Corollary 14.

Results similar to Corollaries 14 and 15 can also be proved for operators of the form $(-\Delta)^{m}+q(x)$, or more generally

$$
\sum_{j=1}^{m} c_{j} \Delta^{j}|x|^{\kappa_{j}} \Delta^{j}+q(x)
$$

The essential ingredient would be to replace Corollary 13 with generalizations of Rellich's inequality [25, p. 90]. One such generalization can be found in a paper of Allegretto [4]. We comment here that Rellich's proof as well as Allegretto's extension can be considerably shortened by using Hardy's inequality [15] on p. 95 of [25] instead of introducing the function $g(r)$. (This removes the restriction $\gamma \leqslant 0$ in Allegretto's extension.) For a rather elegant proof of Rellich's inequality, using Hardy's inequality, and associated references we refer the reader to a paper by Schmincke [29].

REMARK. After the completion of this paper, the authors became aware (through a conversation with A. Kufner) of some interesting new results by B. Opic and A. Kufner [Sobolev weight spaces and the $N$-dimensional Hardy inequality, Trudy Sem. S. L. Soboleva, No. 1 (1983), 108-117 (Russian)] in which the inequality of Lemma 7 is extended to include estimates of the type

$$
\int_{\Omega} a_{0}(x)|\phi(x)|^{p} d x \leqslant c \sum_{i=1}^{n} \int_{\Omega} a_{i}(x)\left|\frac{\partial \phi}{\partial x_{i}}\right|^{p} d x,
$$

for some constant $c>0$ and $p \in(1, \infty)$. Such estimates are sure to lead to a wider variety of applications similar to ones sketched above.

\section{REFERENCES}

1. R. A. Adams, Sobolev spaces, Academic Press, New York, 1975.

2. S. Agmon, Lectures on elliptic boundary-value problems, Van Nostrand, Princeton, N. J., 1965.

3. W. Allegretto, On the equivalence of two types of oscillation for elliptic operators, Pacific J. Math. 55 (1974), 319-328.

4. __ Nonoscillation theory of elliptic equations of order 2n, Pacific J. Math. 64 (1976), 1-16.

5. __ Finiteness of lower spectra of a class of higher order elliptic operators, Pacific J. Math. 83 (1979), 303-309.

6. Spectral estimates and oscillation of singular differential operators, Proc. Amer. Math. Soc. 73 (1979), 51-56.

7. Positive solutions and spectral properties of second order elliptic operators, Pacific J. Math. 92 (1981), 15-25.

8. D. E. Edmunds and W. D. Evans, Spectral theory and embeddings of Sobolev spaces, Quart. J. Math. Oxford Ser. (2) 30 (1979), 431-453. 
9. W. D. Evans, R. T. Lewis, and A. Zettl, Non-self-adjoint operators and their essential spectra, Lecture Notes in Math., vol. 1032, Springer-Verlag, Berlin, 1983, pp. 121-160.

10. L. E. Fraenkel, On the regularity of the boundary in the theory of Sobolev spaces, Proc. London Math. Soc. (3) 39 (1979), 385-427.

11. A. Friedman, Partial differential equations, Holt, Rinehart and Winston, New York, 1969.

12. I. M. Glazman, Direct methods of qualitative spectral analysis of singular differential operators, Israel Program for Scientific Translations, Jerusalem, 1965.

13. T. Kato, Perturbation theory for linear operators, Springer-Verlag, Berlin, 1966.

14. R. T. Lewis, Singular elliptic operators of second-order with purely discrete spectra, Trans. Amer. Math. Soc. 271 (1982), 653-666.

15. , A Friedrichs inequality and an application, Proc. Roy. Soc. Edinburgh Sect. A 97 (1984), 185-191.

16. W. Moss and J. Piepenbrink, Positive solutions of elliptic equations, Pacific J. Math. 75 (1978), 219-226.

17. J. Něcas, Les méthodes directes en théorie des équations elliptiques, Masson, Paris, 1967.

18. A. Persson, Bounds for the discrete part of the spectrum of a semi-bounded Schrödinger operator, Math. Scand. 8 (1960), 143-153.

19. J. Piepenbrink, Finiteness of the lower spectrum of Schrödinger operators, Math. Z. 140 (1974), $29-40$.

20. __ Nonoscillatory elliptic equations, J. Differential Equations 15 (1974), 541-550.

21. A conjecture of Glazman, J. Differential Equations 24 (1977), 173-177.

22. R. Redheffer, On the inequality $\Delta u \geqslant f(u,|\operatorname{grad} u|)$, J. Math. Anal. Appl. 1 (1960), 277-299.

23. M. Reed and B. Simon, Methods of modern mathematical physics. Vol. II, Fourier Analysis, Self-adjointness, Academic Press, New York, 1978.

24. __ Methods of mathematical physics. Vol. IV, Analysis of Operators, Academic Press, New York, 1978.

25. F. Rellich, Perturbation theory of eigenvalue problems, Gordon \& Breach, New York, 1969.

26. M. Schechter, Spectra of partial differential operators, North-Holland, Amsterdam, 1971.

27. __ On the spectra of singular elliptic operators, Mathematika 23 (1976), 107-115.

28. O_ Operator methods in quantum mechanics, North-Holland, Amsterdam, 1981.

29. U.-W. Schmincke, Essential selfadjointness of a Schrödinger operator with strongly singular potential, Math. Z. 124 (1972), 47-50.

30. , The lower spectrum of Schrödinger operators, Arch. Rational Mech. Anal. 75 (1981), $147-155$.

31. B. Simon, Schrödinger semigroups, Bull. Amer. Math. Soc. (N.S.) 7 (1982), 447-526.

32. E. M. Stein, Singular integrals and differentiability properties of functions, Princeton Univ. Press, Princeton, N. J., 1970.

Department of Pure Mathematics, University College, Cardiff CF1 1 XL, Wales, United KINGDOM

Department of Mathematics, University of Alabama at Birmingham, Birmingham, Alabama 35294 